\title{
Controlled Chain Walking for the Synthesis of Thermoplastic Polyolefin Elastomers: Synthesis, Structure, and Properties
}

Kyle S. O’Connor ${ }^{\dagger}$, Annabelle Watts $^{\ddagger}$, Tulaza Vaidya ${ }^{\dagger}$, Anne M. LaPointe ${ }^{\dagger}$, Marc A. Hillmyer*‡, Geoffrey W. Coates*†

${ }^{\dagger}$ Department of Chemistry and Chemical Biology, Baker Laboratory, Cornell University, Ithaca, New York 14853-1301, United States

‡Department of Chemistry, University of Minnesota, Minneapolis, Minnesota 55455-0431, United States

Table of Contents

1. General Considerations $\quad$ S2

$\begin{array}{ll}\text { 2. Materials } & \text { S3 }\end{array}$

3. Complex Synthesis $\quad$ S3

4. Synthesis of Block Copolymers $\quad$ S4

5. Synthesis of Statistical Copolymers $\quad$ S5

6. Casting Polymer Films $\quad$ S5

7. Mechanical Studies and Additional Experiments S6

8. Elastomer GPC and DSC Data $\quad$ S11

$\begin{array}{ll}\text { 9. Ethylene Homopolymerization } & \text { S27 }\end{array}$

10. Determination of Branching Content in Polymer Samples S28

11. ${ }^{1}$ H NMR Spectra of Representative Samples $\quad$ S29

12. ${ }^{13} \mathrm{C}$ NMR Spectra of Representative Samples $\quad$ S31

13. Tensile Strength and Hysteresis Curves for Representative Samples $\quad$ S34

14. DSC Evidence for Strain-Induced Crystallization S39

$\begin{array}{ll}\text { 15. References } & \text { S40 }\end{array}$ 


\section{General Considerations}

Air and/or moisture sensitive compounds were manipulated under an atmosphere of nitrogen using standard schlenk techniques or an MBraun Labmaster glovebox. Flash column chromatography was performed using silica gel (particle size 40-64 $\mu \mathrm{m}, 230-400 \mathrm{mesh}$ ).

The ${ }^{1} \mathrm{H}$ NMR, ${ }^{13} \mathrm{C}\left\{{ }^{1} \mathrm{H}\right\}$ NMR spectra were recorded on Varian INOVA 500, Varian INOVA 600 , or Varian INOVA 400 using the residual non-deuterated solvent signal as a reference. Polymers were analyzed using quantitative ${ }^{1} \mathrm{H}$ and ${ }^{13} \mathrm{C}$ NMR spectroscopy in $\mathrm{Cl}_{2} \mathrm{CDCDCl}_{2}\left(\mathrm{~d}_{2}\right.$ TCE) at $135^{\circ} \mathrm{C} \cdot \mathrm{Cr}(\mathrm{acac})_{3}(\mathrm{acac}=$ acetylacetonate) from Sigma Aldrich was added for quantitative ${ }^{13} \mathrm{C}$ NMR analysis of select samples $\left(17 \mathrm{mg}\right.$ of $\mathrm{Cr}(\mathrm{acac})_{3}$ in $0.5 \mathrm{~mL}$ of $\mathrm{d}_{2}$-TCE, $\left.0.1 \mathrm{M}\right) .{ }^{1}$ MestReNova software was used to process the NMR spectra.

High temperature gel permeation chromatography (GPC) was performed on Agilent PL-GPC 220 equipped with a refractive index (RI) detector and three PL-Gel Mixed B columns. GPC columns were eluted at $1.0 \mathrm{~mL} / \mathrm{min}$ with 1,2,4-trichlorobenzene (TCB) containing $0.01 \mathrm{wt}$ \% ditert-butylhydroxytoluene (BHT) at $150{ }^{\circ} \mathrm{C}$. The samples were prepared in TCB (with BHT) at a concentration of $1.0 \mathrm{mg} / \mathrm{mL}$ and heated at $150{ }^{\circ} \mathrm{C}$ for at least 1 hour prior to injection. GPC data calibration was done with monomodal polyethylene standards from Polymer Standards Service and Agilent.

Differential scanning calorimetry (DSC) measurements were performed on Mettler-Toledo Polymer DSC instrument equipped with an automated sampler. Polymer samples in crimped aluminum pans were analyzed under nitrogen at a heating rate of $10{ }^{\circ} \mathrm{C} / \mathrm{min}$ from -70 to $200{ }^{\circ} \mathrm{C}$. STARe software was used to process the collected data and melting points $\left(T_{\mathrm{m}}\right)$ were obtained and reported from the second heating run. 


\section{Materials}

Acenapthenequinone (Sigma), triphenyl phosphite (Sigma), zinc chloride (Strem), nickel bromide dimethoxyethane adduct (Strem), palladium acetate (Acros), 1-napthylamine (AK Scientific), silver acetate (AK Scientific), potassium hydroxide (Macron), acetic acid (Macron), 2picolinic acid (Alfa Aesar), potassium oxalate (Alfa Aesar), 4-iodobenzotrifluoride (Oakwood Chemical) and pyridine (Fisher Scientific) were used as received.

Anhydrous chlorobenzene was purchased from Sigma Aldrich, sparged with nitrogen for 40 minutes and stored over activated $4 \AA$ molecular sieves. 1-Decene was purchased from Acros Chemicals (95\% purity), distilled and stored over activated $4 \AA$ molecular sieves prior to use. Ethylene was from Airgas, Inc. Methylaluminoxane (MAO) was generously donated by Albemarle Corporation (30 wt $\%$ in toluene) and dried by removing volatiles (toluene and trace trialkylaluminum) under vacuum and heating at $40{ }^{\circ} \mathrm{C}$ for at least 8 hours. Anhydrous toluene, hexanes, and dichloromethane (HPLC) were purchased from Fisher Scientific, sparged with nitrogen for 40 minutes, and purified over solvent columns. NMR solvents were purchased from Cambridge Isotope Laboratories and stored over activated $4 \AA$ A molecular sieves.

\section{Complex Synthesis}

Complex 2 was synthesized according to literature procedures. ${ }^{2}$ The resulting dark red crystals were stored in a vial inside a glovebox and crushed into a fine powder immediately before use.

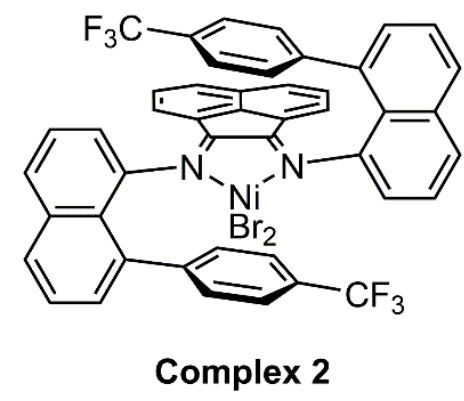




\section{General Polymerization Scheme (Synthesis of Block Copolymers)}

All polymerizations were set up in a MBraun Labmaster glovebox. An oven-dried $200 \mathrm{~mL}$ Fisher-Porter bottle (Andrews Glass) equipped with a magnetic stir bar was charged with MAO (200 eq, $6.79 \mathrm{mmol})$, solvent (72 $\mathrm{mL}$ of toluene or chlorobenzene) and 1-decene $(0.1 \mathrm{M}, 1.6 \mathrm{~mL}$ or $0.2 \mathrm{M}, 3.2 \mathrm{~mL}$ ). The vessel was sealed with a Swagelok reactor head. Complex 2 (1 eq, 34.1 $\mu \mathrm{mol})$ was dissolved in chlorobenzene $(6 \mathrm{~mL})$ and drawn into a gas tight syringe equipped with a stainless steel needle, then sealed at the tip using a rubber septum. The vessel and syringe were removed from the glovebox. The vessel was submerged in a water bath, connected to a nitrogen inlet and pressurized to $20 \mathrm{psig}$ (1.4 atm). Under this pressure, the catalyst solution was injected. The polymerization was run for the appropriate time to grow the first hard block from 1-decene. An aliquot was removed $(8 \mathrm{~mL})$ and precipitated into acidic methanol $(5 \% \mathrm{HCl} \mathrm{v} / \mathrm{v}, \sim 50 \mathrm{ml})$ for future analysis. The nitrogen atmosphere was then exchanged with ethylene (16 psig, $1.1 \mathrm{~atm})$ by cycling ethylene into the bottle and releasing pressure through the top valve via a syringe needle inserted through a rubber septum at least 3 times. The ethylene inlet was left open for a given amount of time to grow the soft block, after which the ethylene atmosphere was replaced with nitrogen by cycling nitrogen into the bottle and releasing pressure through the top valve at least 8 times. An aliquot was removed $(3 \mathrm{~mL})$ and precipitated into acidic methanol for future analysis. For triblock copolymers, the polymerization was quenched after the given time by reducing pressure through the top valve and injecting $10 \mathrm{~mL}$ of methanol into the vessel with vigorous stirring. The polymer solution was then precipitated into a solution of acidic methanol $(5 \% \mathrm{HCl}$ $\mathrm{v} / \mathrm{v} \sim 500 \mathrm{~mL}$ ), and stirred for at least 4 hours. All polymers were filtered, washed with methanol and dried under vacuum at $45^{\circ} \mathrm{C}$ until constant weight. For higher order block copolymers, additional cycles were repeated until the desired block architecture was achieved. 


\section{Synthesis of Statistical Copolymers}

An oven-dried $200 \mathrm{~mL}$ Fisher-Porter bottle equipped with a magnetic stir bar was charged with MAO (200 eq, $6.79 \mathrm{mmol}$ ), appropriate solvent (72 $\mathrm{mL}$ of toluene or chlorobenzene) and 1-decene (0.2 M, $3.2 \mathrm{~mL})$. Complex 2 was dissolved in chlorobenzene $(6 \mathrm{~mL})$ and drawn into a gas tight syringe equipped with a stainless steel needle, sealed at the tip using a rubber septum. The vessel and syringe were removed from the glovebox. The vessel was submerged in a water bath and connected to an ethylene inlet (1.1 atm), cycling 3 times to exchange the nitrogen environment. While open to ethylene, the catalyst was immediately injected into the reaction vessel. After the desired polymerization time, the pressure was reduced through the top valve and the reaction mixture was quenched with methanol $(10 \mathrm{~mL})$ under vigorous stirring. The polymer solution was precipitated into acidic methanol $(5 \% \mathrm{HCl} \mathrm{v} / \mathrm{v}, \sim 500 \mathrm{~mL})$, and stirred for at least 4 hours. The resulting polymers were filtered, washed with methanol and dried in vacuo until constant weight.

\section{Casting Polymer Films}

All polymer samples were melt-casted using a Carver Press hot plate. Each sample was loaded into a rectangular stainless steel mold (92 mm x $30 \mathrm{~mm}$ x $0.5 \mathrm{~mm}$ ) sandwiched between two Mylar sheets and two stainless steel sheets and pressed under a pressure of $\sim 52 \mathrm{MPa}$ at $110{ }^{\circ} \mathrm{C}$ for 15 minutes. At this pressure, the sample was cooled to $22{ }^{\circ} \mathrm{C}$ at a rate of $6{ }^{\circ} \mathrm{C} / \mathrm{min}$ over a period of 20 minutes. The rectangular film was removed from the mold and excess polymer around the edges was removed with a razor blade. The film was subsequently cut into tensile bars using a stainless steel polymer die (gauge length $=16 \mathrm{~mm}$, gauge width $=3 \mathrm{~mm}$, gauge thickness $=0.6 \mathrm{~mm}$ ) to give polymer bars which were rubbery, clear and easy to handle. 


\section{Mechanical Studies and Additional Experiments}

Mechanical studies were performed using a Shimadzu Autograph AGS-X Series tensile tester. For tensile strength and hysteresis experiments, a crosshead velocity of $50 \mathrm{~mm} / \mathrm{min}$ was used for each sample. For tensile strength experiments, tensile bars were elongated until break. At least five tensile bars were tested for each individual sample. For hysteresis experiments, tensile bars were cycled ten times to $300 \%$ strain. Three tensile bars were tested for each individual sample.

A creep experiment was performed on samples with various block architectures in order to probe their resistance to permanent deformation. Tensile bars were elongated to $300 \%$ strain and held at a constant stress over a period of three hours. The change in strain over time was monitored. Individual samples required different levels of stress in order to achieve the initial $300 \%$ strain (Figure S1).

Figure S1. Creep Experiment for Various Block Structures.

\begin{tabular}{cc}
\hline Sample (Solvent) & $\begin{array}{c}\text { Stress } \\
(\mathbf{M P a})\end{array}$ \\
\hline Statistical $(\mathrm{PhCl})$ & 1.8 \\
Statistical $(\mathrm{PhMe})$ & 2.4 \\
Diblock $(\mathrm{PhCl})$ & 4.0 \\
Triblock $(\mathrm{PhCl})$ & 4.5 \\
Pentablock $(\mathrm{PhCl})$ & 5.8 \\
\hline
\end{tabular}

Tensile bars after prolonged stress Top $=$ Diblock

Bottom $=$ Triblock

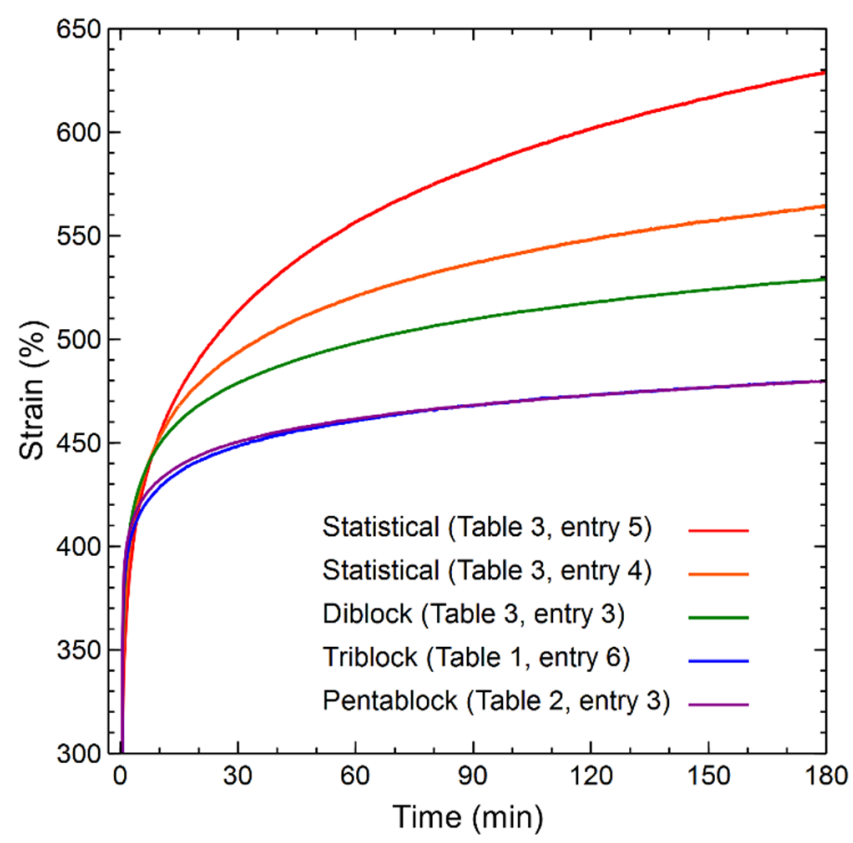


An additional creep experiment was performed where samples were subjected to the same constant force $(1.8 \mathrm{~N})$ over a period of three hours, monitoring change in strain over time. A similar trend was found under these conditions, where lower block structures (diblock) deformed more rapidly than higher block structures (pentablock).

Figure S2. Creep Experiment at Constant Force for Diblock and Pentablock Copolymers

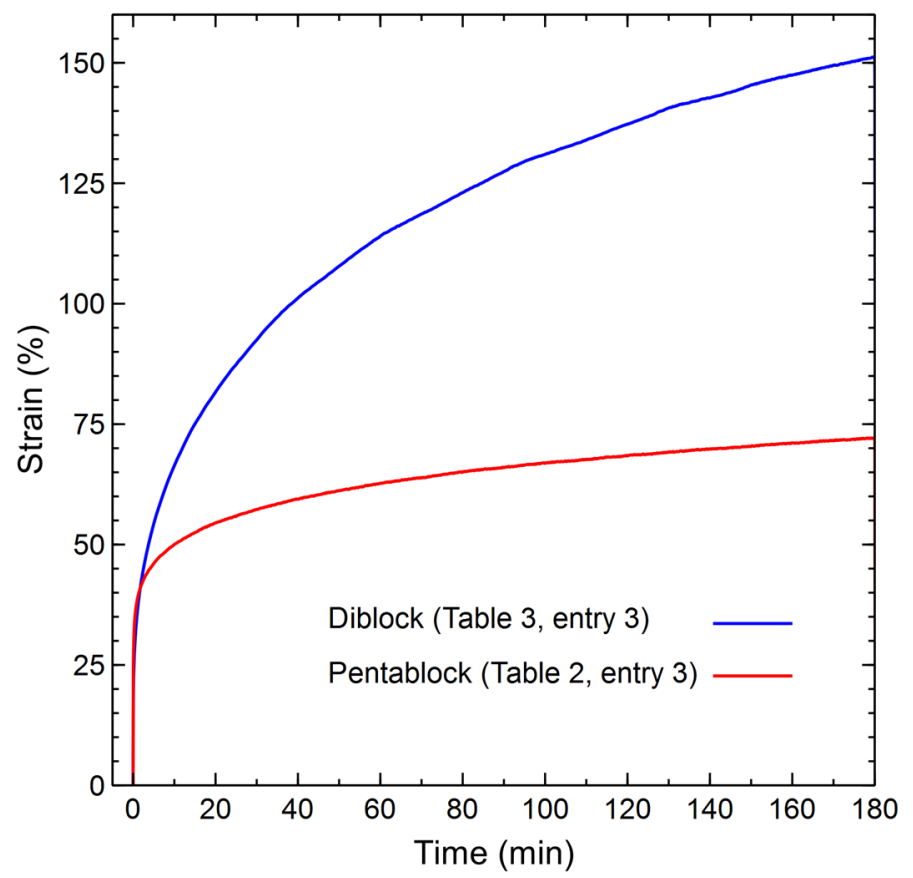


Two samples were further analyzed to determine the dependence of hysteresis rate on elastic return properties. A representative triblock (Table 1, Entry 6) and statistical (Table 3, Entry 5) copolymer were stretched to $300 \%$ strain at $5 \mathrm{~mm} / \mathrm{min}$ over 10 cycles (compared to $50 \mathrm{~mm} / \mathrm{min}$ ). It was determined that hysteresis rate does not have a significant effect on elastic return properties over this range (Figure S3 and S4).

Figure S3. Hysteresis Curves at Fast and Slow Elongation Rates for Triblock Copolymer
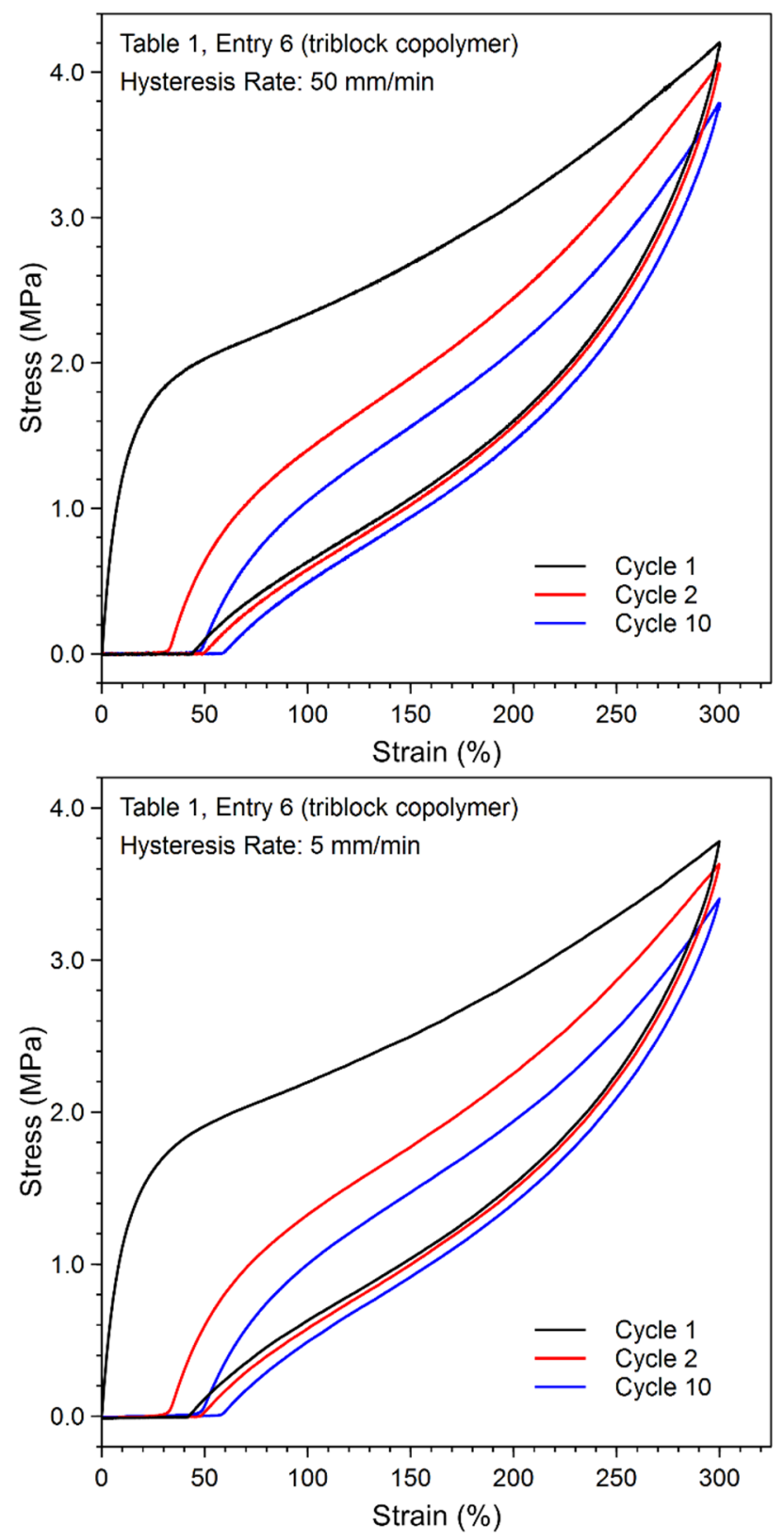
Figure S4. Hysteresis Curves at Fast and Slow Elongation Rates for Statistical Copolymer
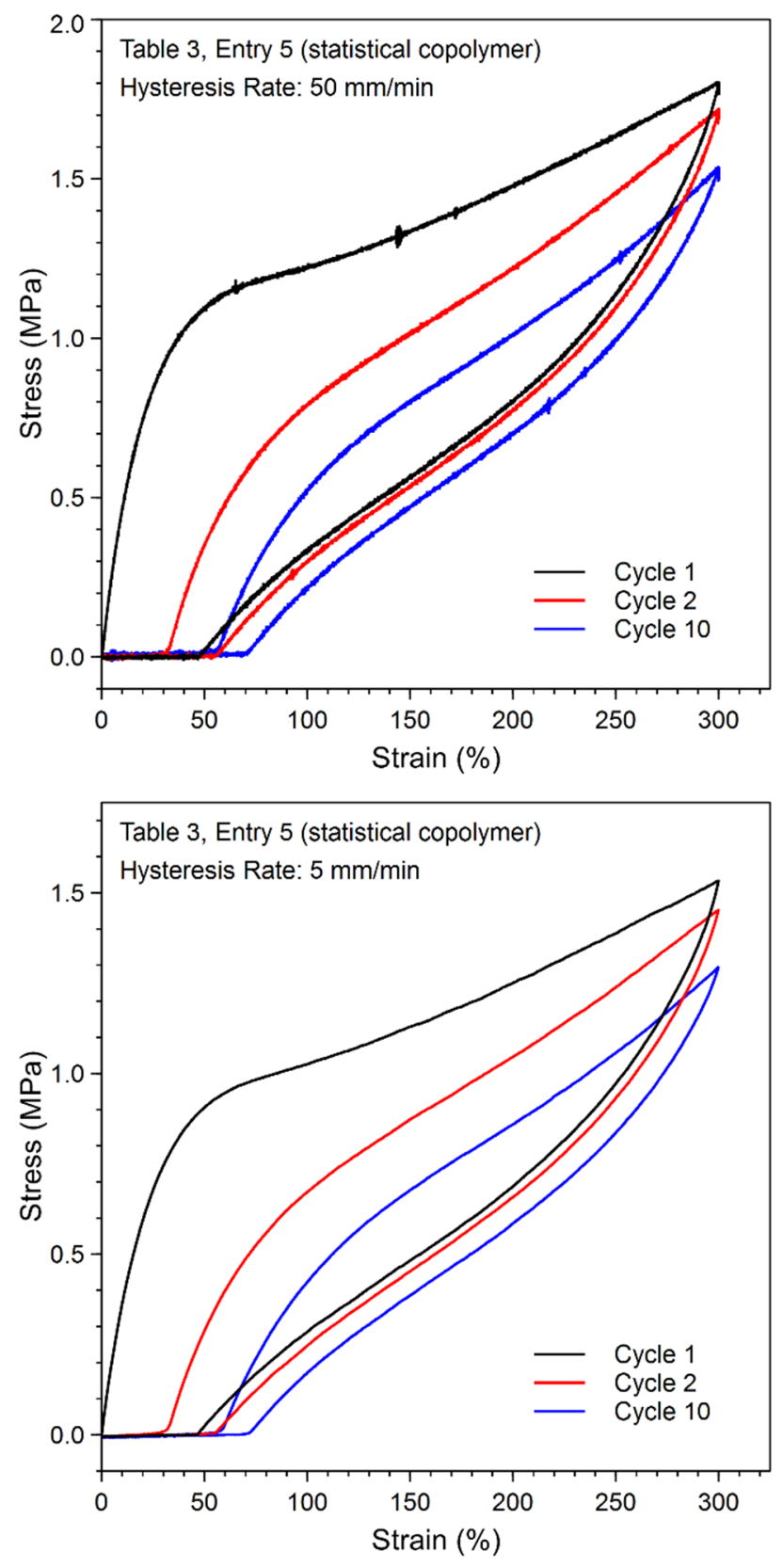
A sample of chain straightened 1-decene was prepared and the mechanical properties were tested in order to determine the behavior of the hard block. The tensile plot below shows that the hard block does not behave as an elastomer, demonstrating a high Young's modulus and plastic deformation. No elastic recovery is observed.

Figure S5. Synthesis, Characterization and Tensile Strength Curve of Hard Block
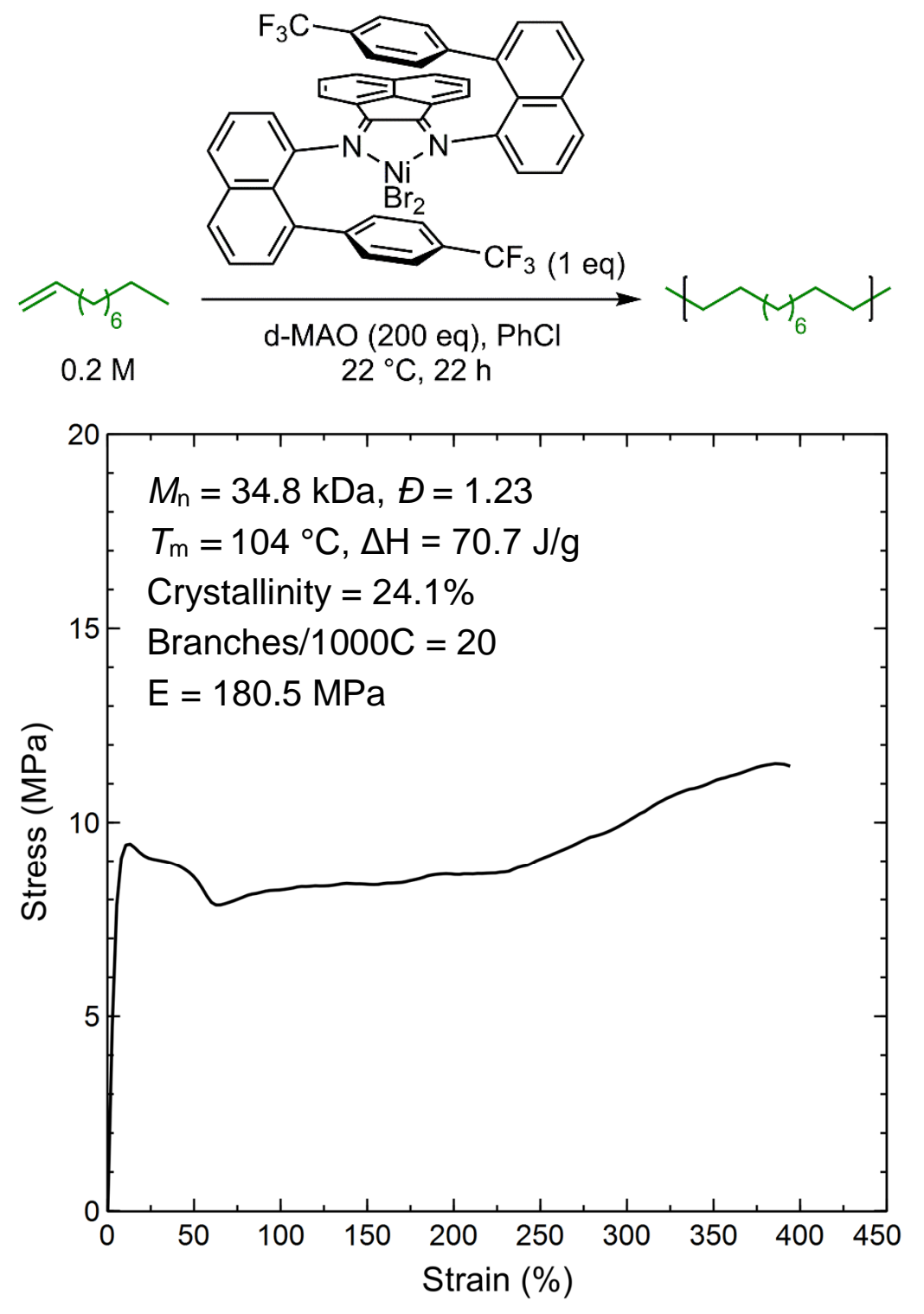


\section{Elastomer GPC and DSC Data}

GPC traces are plotted where lower retention time signifies higher molecular weight (block size increases from right to left). The refractive index (RI) of each aliquot was normalized to 1.0. DSC traces of the second heat of each aliquot are plotted. Sample crystallinity $(X)$ was calculated using the reference enthalpy $\left(\Delta \mathrm{H}_{\mathrm{m}}{ }^{0}=293.6 \mathrm{~J} \mathrm{~g}^{-1}\right)$ for fully crystalline polyethylene: $\left[\left(\Delta \mathrm{H}_{\mathrm{m}}-\Delta \mathrm{H}_{\mathrm{c}}\right)\right.$ / $\left.\Delta \mathrm{H}_{\mathrm{m}}{ }^{0}\right] \times 100$. The glass transition temperature $\left(T_{\mathrm{g}}\right)$ for each block could not be visualized over the given temperature range $\left(-70\right.$ to $\left.200{ }^{\circ} \mathrm{C}\right)$, which is consistent with polyethylene possessing a very low $T_{\mathrm{g}}{ }^{3}$

Additional data tables are included below the GPC and DSC trace for each sample. Yield of polymer is determined from the aliquot removed during the polymerization. Branches per 1000 carbons were determined by ${ }^{1} \mathrm{H}$ NMR for the final block copolymer of each sample, as well as select aliquots (refer to page S28 for equation). 
Triblock Copolymer (Table 1, Entry 1)
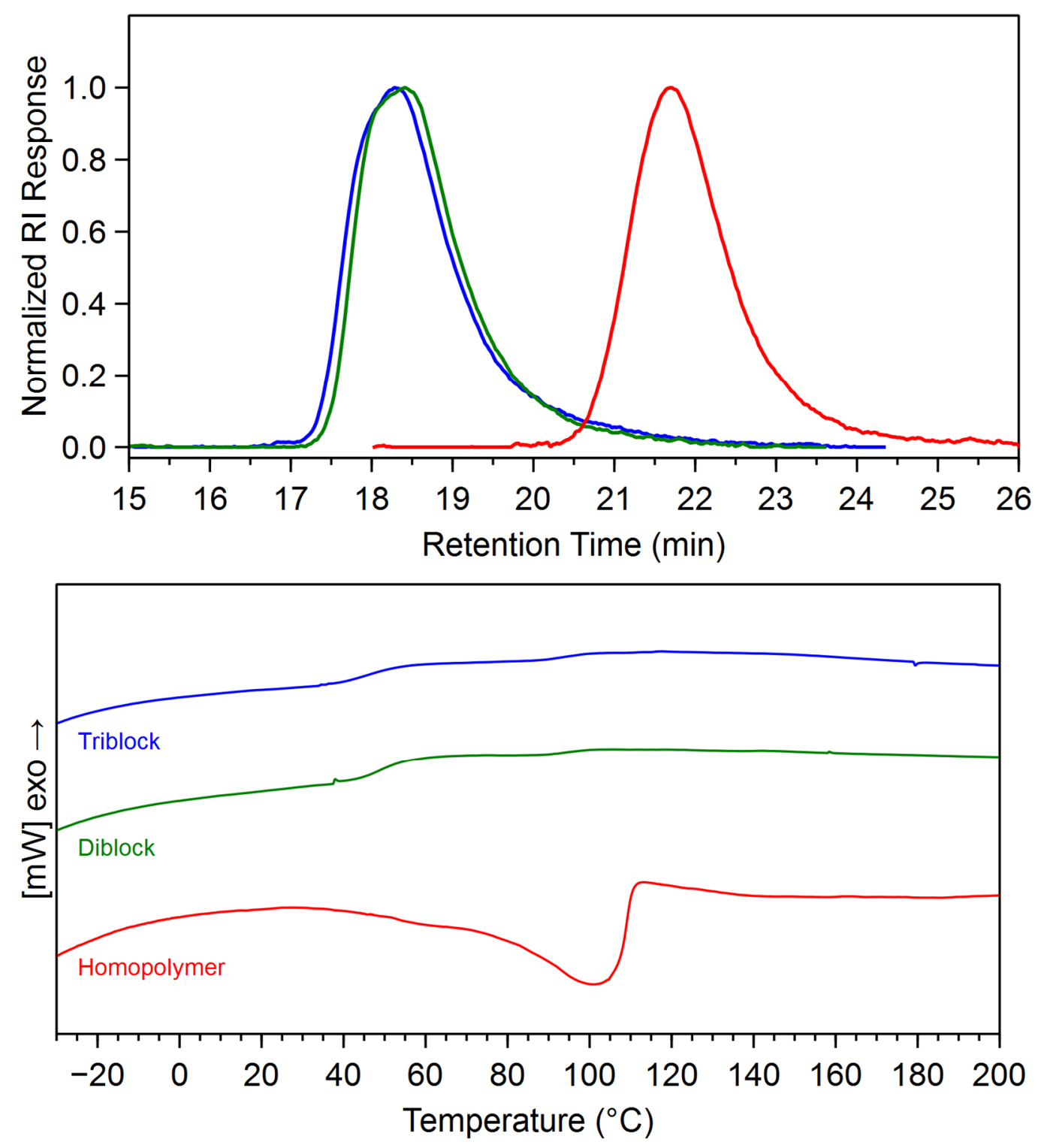

\begin{tabular}{ccccccccc}
\hline Sample & $\begin{array}{c}\text { Time } \\
(\mathbf{h})\end{array}$ & $\begin{array}{c}\text { Yield } \\
(\mathbf{g})\end{array}$ & $\begin{array}{c}\boldsymbol{M}_{\mathbf{n}} \\
(\mathbf{k D a})\end{array}$ & $\boldsymbol{D}$ & $\begin{array}{c}\boldsymbol{T}_{\mathbf{m}} \\
\left({ }^{\circ} \mathbf{C}\right)\end{array}$ & $\begin{array}{c}\boldsymbol{\Delta H} \\
(\mathbf{J} / \mathbf{g})\end{array}$ & $\begin{array}{c}\boldsymbol{X} \\
(\boldsymbol{\%})\end{array}$ & $\begin{array}{c}\text { Branches/ } \\
\mathbf{1 0 0 0 C}\end{array}$ \\
\hline Homopolymer & 2.0 & 0.014 & 4.9 & 1.28 & 101 & 103.0 & 35.1 & $\mathrm{n} / \mathrm{d}$ \\
Diblock & 0.8 & 0.048 & 94.4 & 1.34 & 43,89 & $3.1,1.4$ & $1.0,0.5$ & $\mathrm{n} / \mathrm{d}$ \\
Triblock & 4.5 & 1.64 & 99.2 & 1.42 & 40,89 & $1.9,1.2$ & $0.6,0.4$ & 69 \\
\hline
\end{tabular}


Triblock Copolymer GPC/DSC (Table 1, Entry 2)
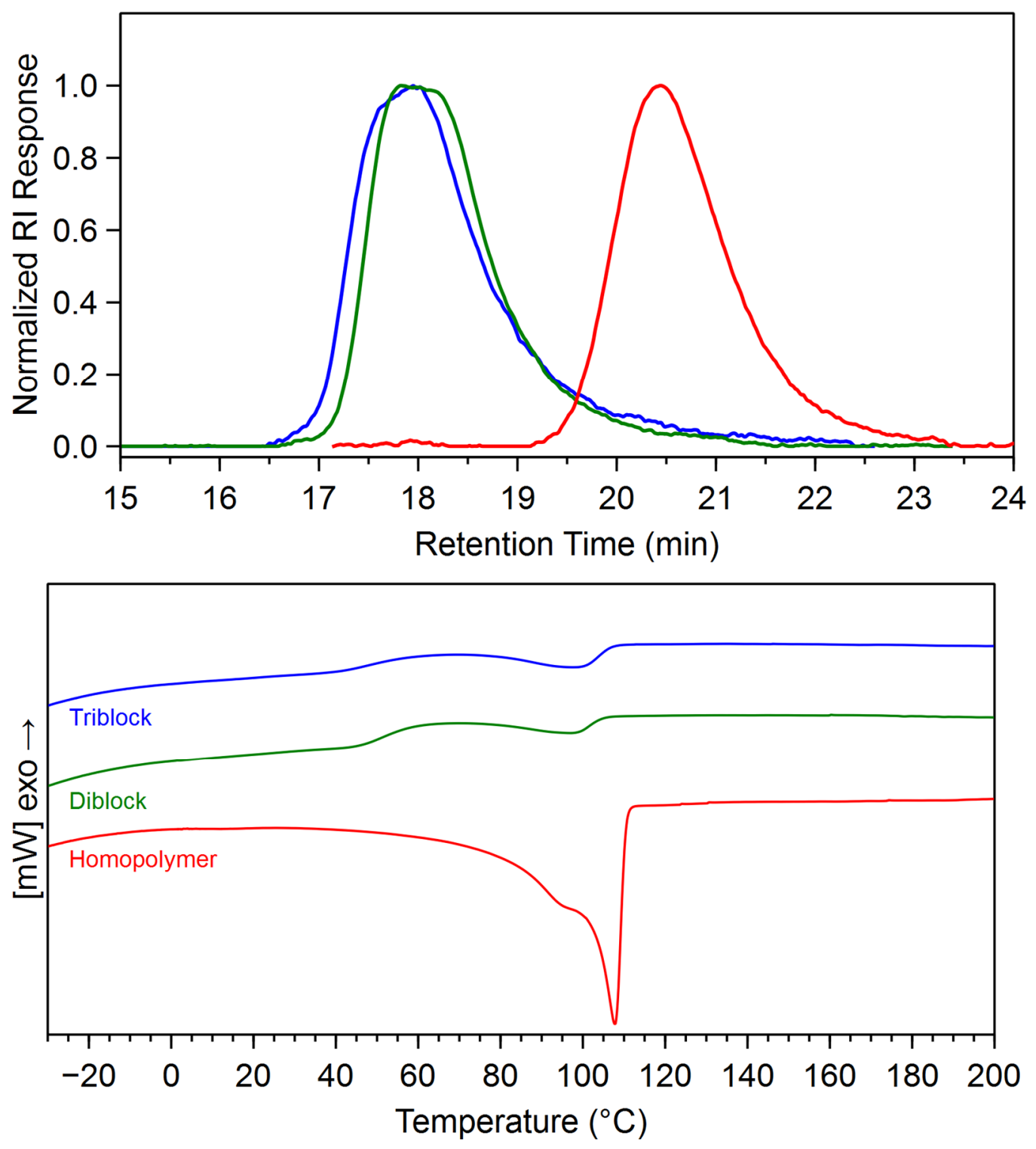

\begin{tabular}{ccccccccc}
\hline Sample & $\begin{array}{c}\text { Time } \\
(\mathbf{h})\end{array}$ & $\begin{array}{c}\text { Yield } \\
(\mathbf{g})\end{array}$ & $\begin{array}{c}\boldsymbol{M}_{\mathbf{n}} \\
(\mathbf{k D a})\end{array}$ & $\boldsymbol{D}$ & $\begin{array}{c}\boldsymbol{T}_{\mathbf{m}} \\
\left({ }^{\circ} \mathbf{C}\right)\end{array}$ & $\begin{array}{c}\Delta \mathbf{H} \\
(\mathbf{J} / \mathbf{g})\end{array}$ & $\begin{array}{c}\boldsymbol{X} \\
(\boldsymbol{\%})\end{array}$ & $\begin{array}{c}\text { Branches/ } \\
\mathbf{1 0 0 0 C}\end{array}$ \\
\hline Homopolymer & 5.5 & 0.014 & 12.1 & 1.32 & 108 & 106.0 & 36.1 & $\mathrm{n} / \mathrm{d}$ \\
Diblock & 0.8 & 0.060 & 107.1 & 1.36 & 46,97 & $4.1,7.7$ & $1.4,2.6$ & $\mathrm{n} / \mathrm{d}$ \\
Triblock & 16.5 & 2.08 & 116.9 & 1.42 & 42,99 & $1.8,10.6$ & $0.6,3.6$ & 65 \\
\hline
\end{tabular}


Triblock Copolymer GPC/DSC (Table 1, Entry 3)
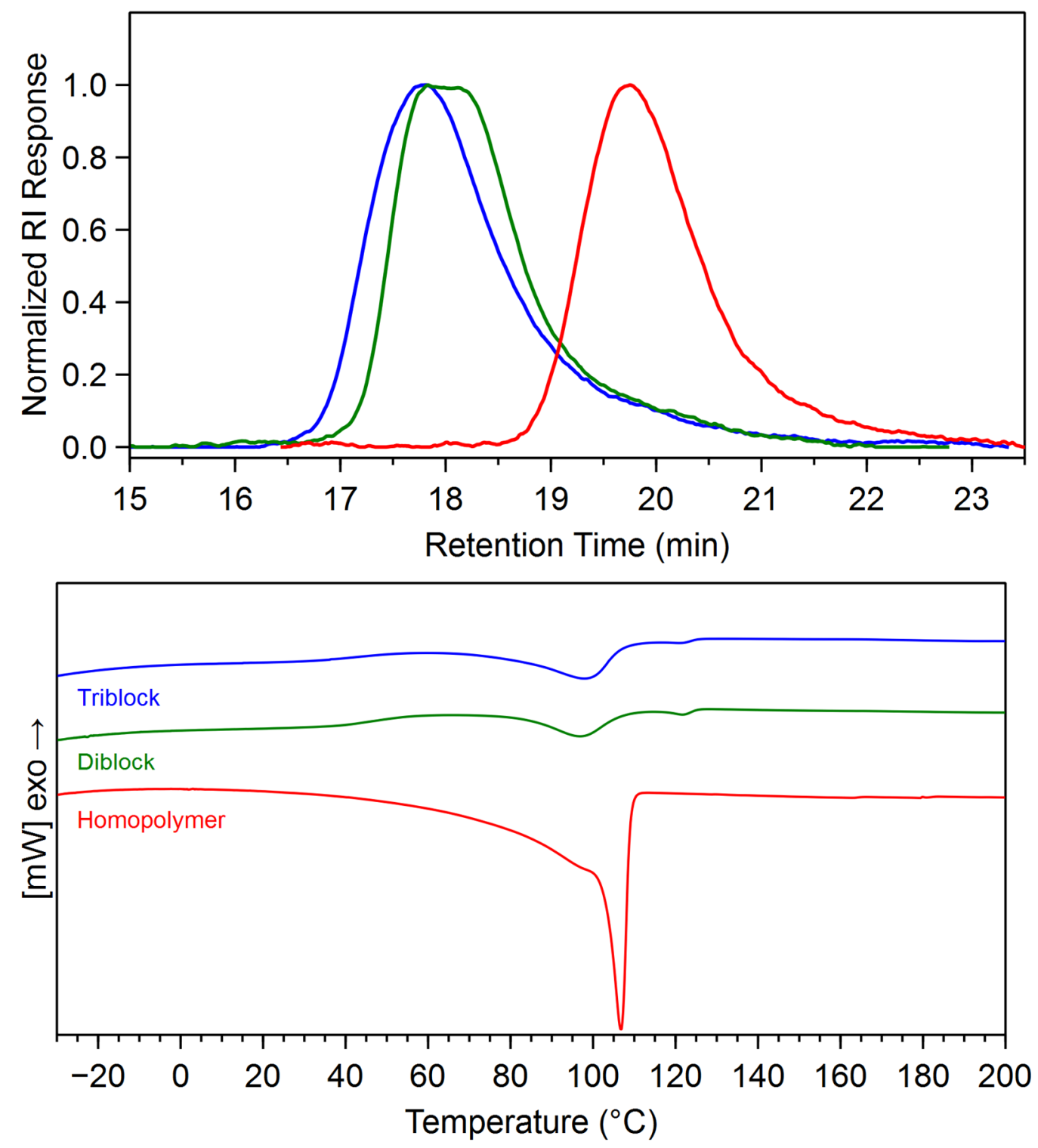

\begin{tabular}{ccccccccc}
\hline Sample & $\begin{array}{c}\text { Time } \\
(\mathbf{h})\end{array}$ & $\begin{array}{c}\text { Yield } \\
(\mathbf{g})\end{array}$ & $\begin{array}{c}\boldsymbol{M}_{\mathbf{n}} \\
(\mathbf{k D a})\end{array}$ & $\boldsymbol{D}$ & $\begin{array}{c}\boldsymbol{T}_{\mathbf{m}} \\
\left({ }^{\mathbf{C}} \mathbf{C}\right)\end{array}$ & $\begin{array}{c}\Delta \mathbf{H} \\
(\mathbf{J} / \mathbf{g})\end{array}$ & $\begin{array}{c}\boldsymbol{X} \\
(\boldsymbol{\%})\end{array}$ & $\begin{array}{c}\text { Branches/ } \\
\mathbf{1 0 0 0 C}\end{array}$ \\
\hline Homopolymer & 15.0 & 0.031 & 22.1 & 1.34 & 107 & 90.6 & 30.9 & $\mathrm{n} / \mathrm{d}$ \\
Diblock & 0.9 & 0.062 & 95.4 & 1.48 & 40,97 & $0.9,11.4$ & $0.3,3.9$ & $\mathrm{n} / \mathrm{d}$ \\
Triblock $^{*}$ & 33.0 & 2.49 & 113.2 & 1.55 & 98 & 19.4 & 6.6 & 57 \\
\hline${ }^{*}$ Addition
\end{tabular}

*Additional 1-decene $(0.81 \mathrm{~mL})$ was injected via gas-tight syringe into reaction in order to have enough free monomer to complete the polymerization. 
Triblock Copolymer GPC/DSC (Table 1, Entry 4)
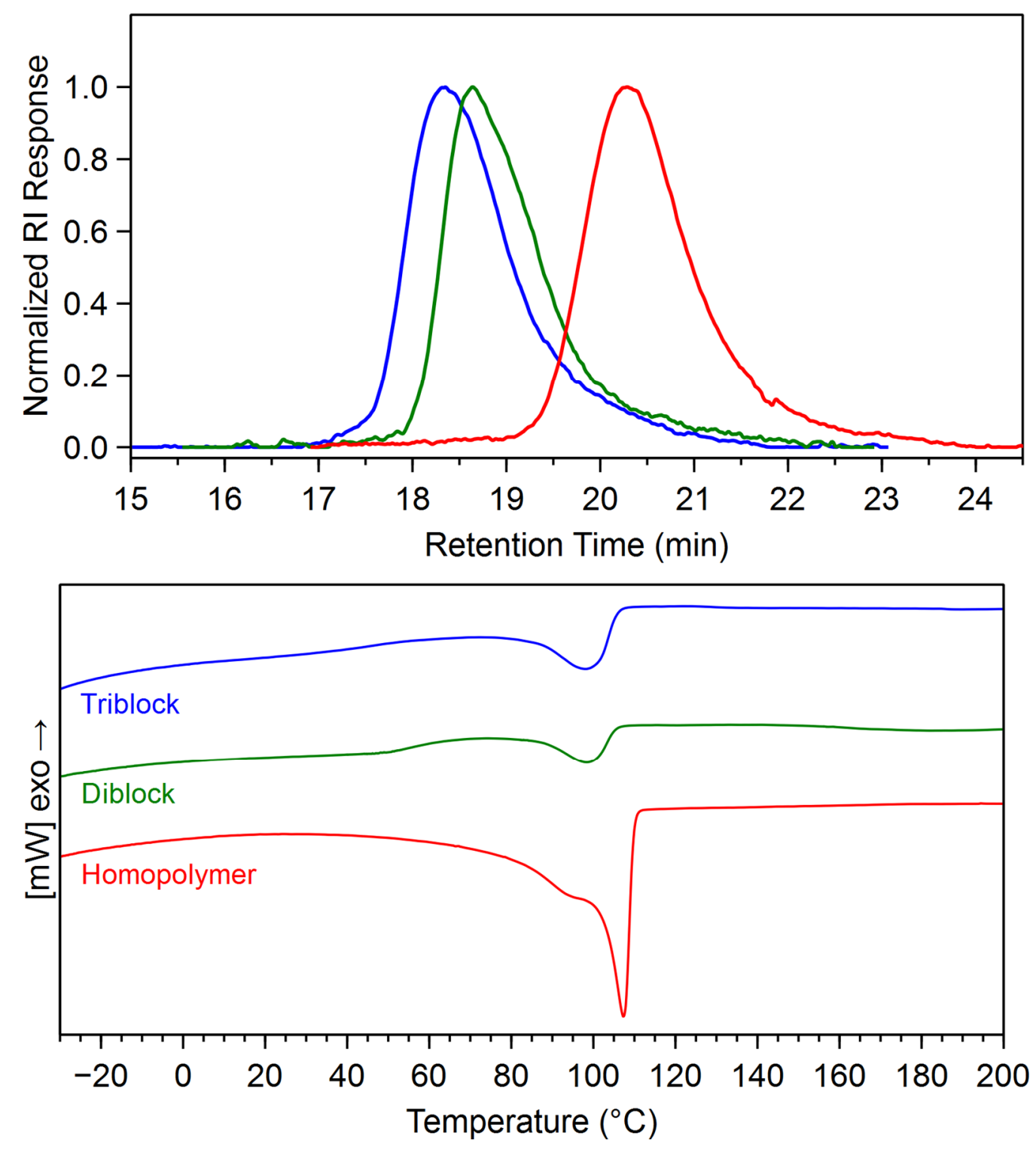

\begin{tabular}{ccccccccc}
\hline Sample & $\begin{array}{c}\text { Time } \\
(\mathbf{h})\end{array}$ & $\begin{array}{c}\text { Yield } \\
(\mathbf{g})\end{array}$ & $\begin{array}{c}\boldsymbol{M}_{\mathbf{n}} \\
(\mathbf{k D a})\end{array}$ & $\boldsymbol{D}$ & $\begin{array}{c}\boldsymbol{T}_{\mathbf{m}} \\
\left({ }^{\circ} \mathbf{C}\right)\end{array}$ & $\begin{array}{c}\Delta \mathbf{H} \\
(\mathbf{J} / \mathbf{g})\end{array}$ & $\begin{array}{c}\boldsymbol{X} \\
(\boldsymbol{\%})\end{array}$ & $\begin{array}{c}\text { Branches/ } \\
\mathbf{1 0 0 0 C}\end{array}$ \\
\hline Homopolymer & 7.5 & 0.010 & 14.0 & 1.30 & 107 & 114.8 & 39.1 & $\mathrm{n} / \mathrm{d}$ \\
Diblock & 0.5 & 0.023 & 56.7 & 1.29 & 51,99 & $2.3,17.2$ & 0.85 .8 & $\mathrm{n} / \mathrm{d}$ \\
Triblock & 24.0 & 1.34 & 73.9 & 1.38 & 99 & 20.4 & 6.9 & 60 \\
\hline
\end{tabular}


Triblock Copolymer GPC/DSC (Table 1, Entry 5)
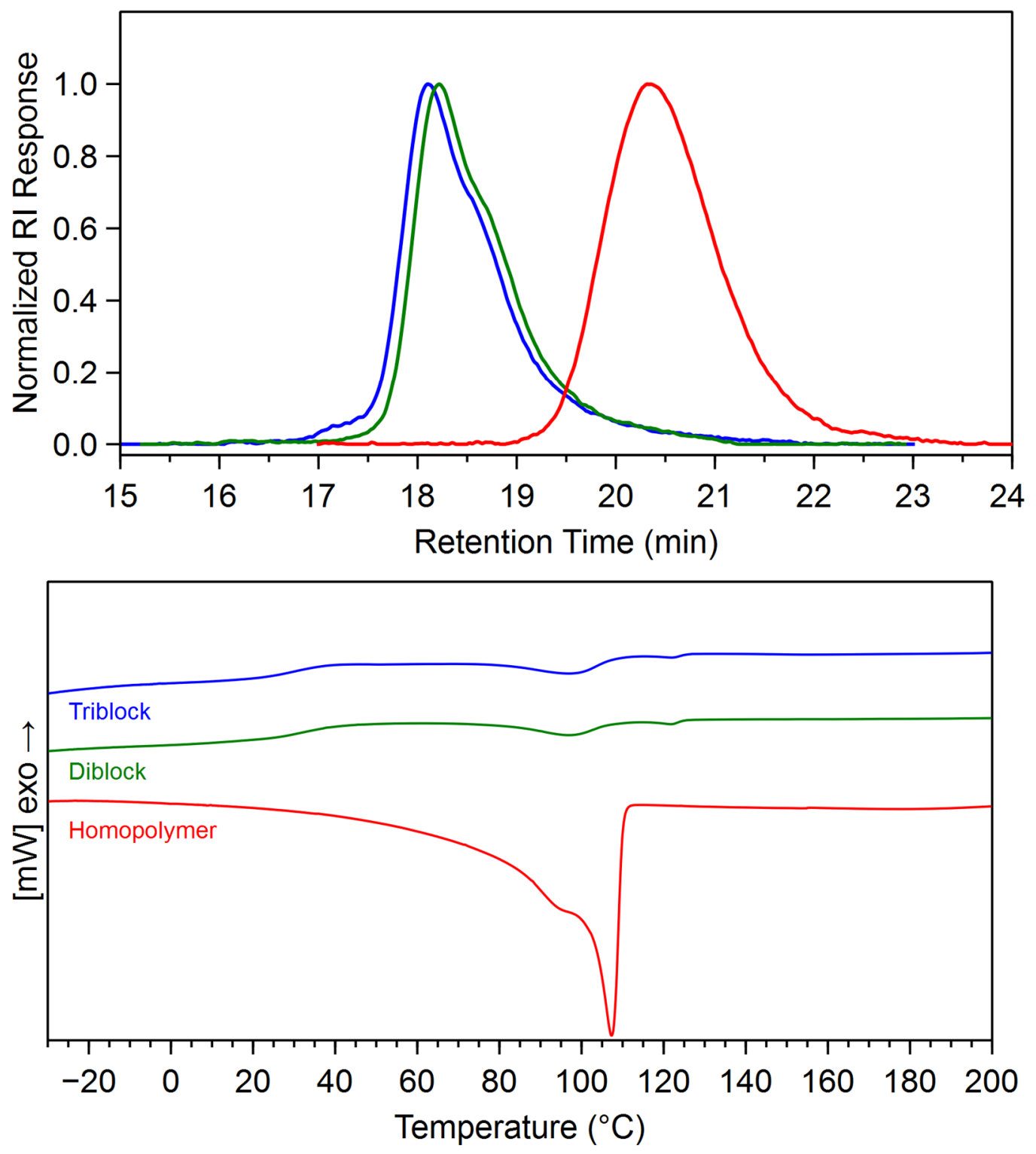

\begin{tabular}{ccccccccc}
\hline Sample & $\begin{array}{c}\text { Time } \\
(\mathbf{h})\end{array}$ & $\begin{array}{c}\text { Yield } \\
(\mathbf{g})\end{array}$ & $\begin{array}{c}\boldsymbol{M}_{\mathbf{n}} \\
(\mathbf{k D a})\end{array}$ & $\boldsymbol{D}$ & $\begin{array}{c}\boldsymbol{T}_{\mathbf{m}} \\
\left({ }^{\circ} \mathbf{C}\right)\end{array}$ & $\begin{array}{c}\Delta \mathbf{H} \\
(\mathbf{J} / \mathbf{g})\end{array}$ & $\begin{array}{c}\boldsymbol{X} \\
(\boldsymbol{\%})\end{array}$ & $\begin{array}{c}\text { Branches/ } \\
\mathbf{1 0 0 0 C}\end{array}$ \\
\hline Homopolymer & 7.0 & 0.041 & 13.7 & 1.29 & 108 & 114.8 & 39.1 & $\mathrm{n} / \mathrm{d}$ \\
Diblock & 1.0 & 0.113 & 79.9 & 1.30 & 27,98 & $2.0,7.1$ & $0.7,2.4$ & $\mathrm{n} / \mathrm{d}$ \\
Triblock & 20.0 & 2.38 & 91.2 & 1.31 & 23,97 & $1.2,7.6$ & $0.4,2.6$ & 74 \\
\hline
\end{tabular}


Triblock Copolymer GPC/DSC (Table 1, Entry 6)
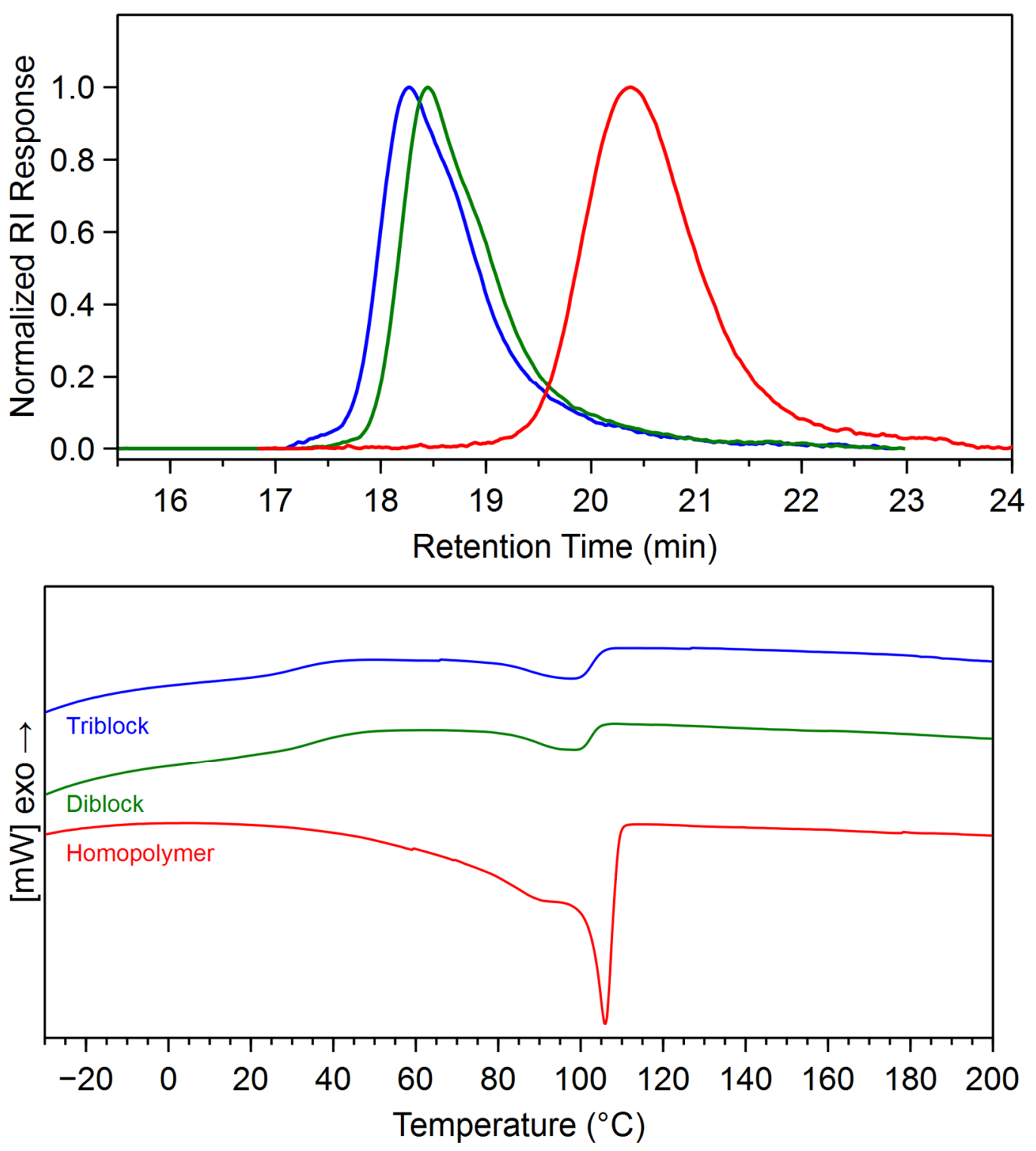

\begin{tabular}{ccccccccc}
\hline Sample & $\begin{array}{c}\text { Time } \\
(\mathbf{h})\end{array}$ & $\begin{array}{c}\text { Yield } \\
(\mathbf{g})\end{array}$ & $\begin{array}{c}\boldsymbol{M}_{\mathbf{n}} \\
(\mathbf{k D a})\end{array}$ & $\boldsymbol{D}$ & $\begin{array}{c}\boldsymbol{T}_{\mathbf{m}} \\
\left({ }^{\circ} \mathbf{C}\right)\end{array}$ & $\begin{array}{c}\Delta \mathbf{H} \\
(\mathbf{J} / \mathbf{g})\end{array}$ & $\begin{array}{c}\boldsymbol{X} \\
(\boldsymbol{\%})\end{array}$ & $\begin{array}{c}\text { Branches/ } \\
\mathbf{1 0 0 0 C}\end{array}$ \\
\hline Homopolymer & 3.0 & 0.028 & 13.4 & 1.29 & 106 & 93.6 & 31.8 & $\mathrm{n} / \mathrm{d}$ \\
Diblock & 0.8 & 0.071 & 66.3 & 1.29 & 30,99 & $1.3,9.7$ & $0.4,3.3$ & $\mathrm{n} / \mathrm{d}$ \\
Triblock & 5.0 & 2.39 & 78.1 & 1.31 & 25,98 & $0.6,12.9$ & $0.2,4.4$ & 70 \\
\hline
\end{tabular}


Pentablock Copolymer GPC/DSC (Table 2, Entry 1)
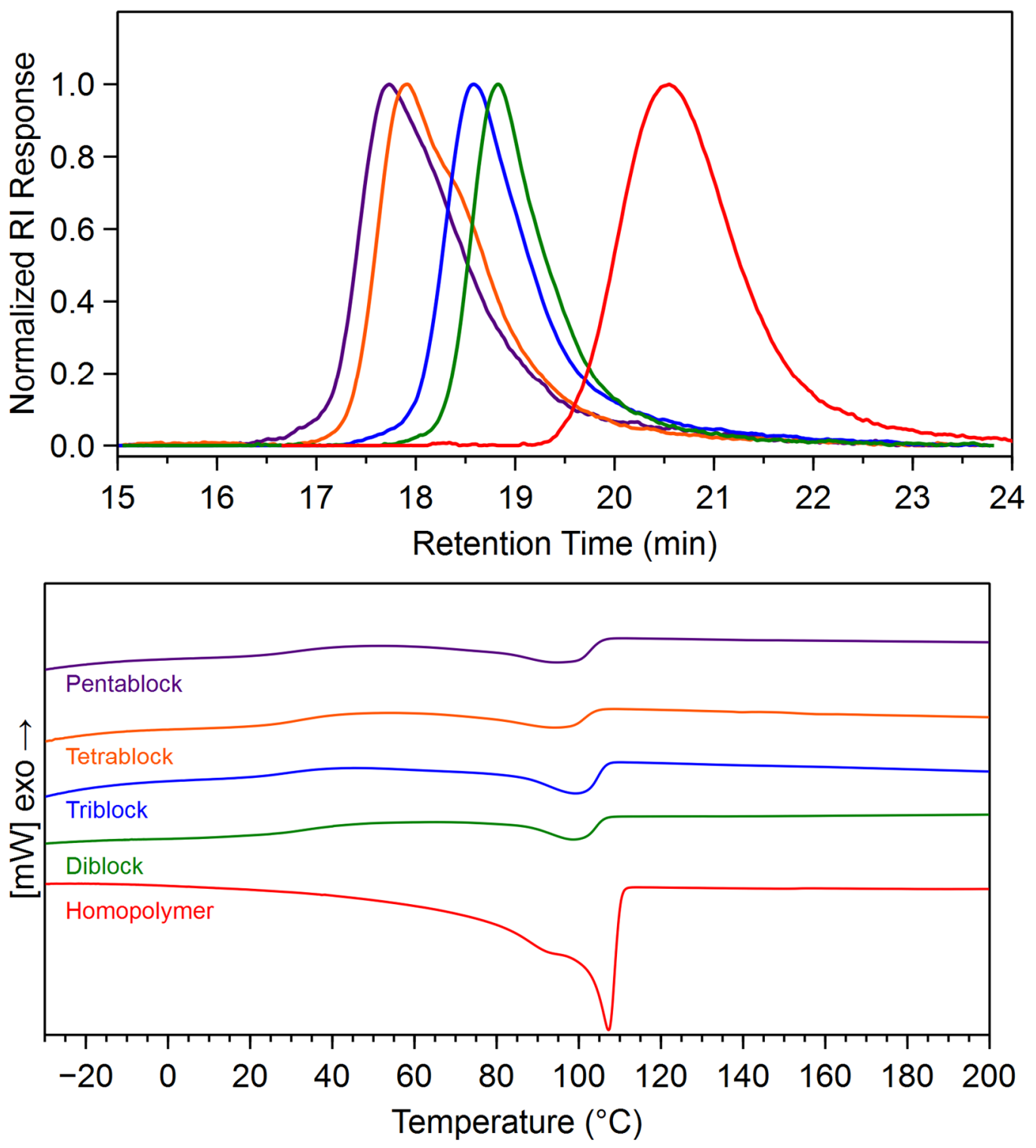

\begin{tabular}{ccccccccc}
\hline Sample & $\begin{array}{c}\text { Time } \\
(\mathbf{h})\end{array}$ & $\begin{array}{c}\text { Yield } \\
(\mathbf{g})\end{array}$ & $\begin{array}{c}\boldsymbol{M}_{\mathbf{n}} \\
(\mathbf{k D a})\end{array}$ & $\boldsymbol{D}$ & $\begin{array}{c}\boldsymbol{T}_{\mathbf{m}} \\
\left({ }^{\circ} \mathbf{C}\right)\end{array}$ & $\begin{array}{c}\Delta \mathbf{H} \\
(\mathbf{J} / \mathbf{g})\end{array}$ & $\begin{array}{c}\boldsymbol{X} \\
(\boldsymbol{\%})\end{array}$ & $\begin{array}{c}\text { Branches/ } \\
\mathbf{1 0 0 0 C}\end{array}$ \\
\hline Homopolymer & 6.0 & 0.017 & 11.2 & 1.32 & 107 & 103.9 & 35.3 & $\mathrm{n} / \mathrm{d}$ \\
Diblock & 0.7 & 0.053 & 51.6 & 1.24 & 28,99 & $0.9,11.5$ & 0.33 .9 & $\mathrm{n} / \mathrm{d}$ \\
Triblock & 14.5 & 0.040 & 57.7 & 1.32 & 24,100 & $0.6,16.6$ & 5.9 & $\mathrm{n} / \mathrm{d}$ \\
Tetrablock & 0.7 & 0.080 & 99.9 & 1.35 & 25,96 & $1.6,12.5$ & 4.8 & $\mathrm{n} / \mathrm{d}$ \\
Pentablock* & 14.5 & 3.25 & 117.0 & 1.38 & 24,98 & $1.2,15.2$ & 5.6 & 65 \\
\hline
\end{tabular}

*Additional 1-decene $(1.0 \mathrm{~mL})$ was injected via gas-tight syringe into reaction in order to have enough free monomer to complete the polymerization. 
Pentablock Copolymer GPC/DSC (Table 2, Entry 2)
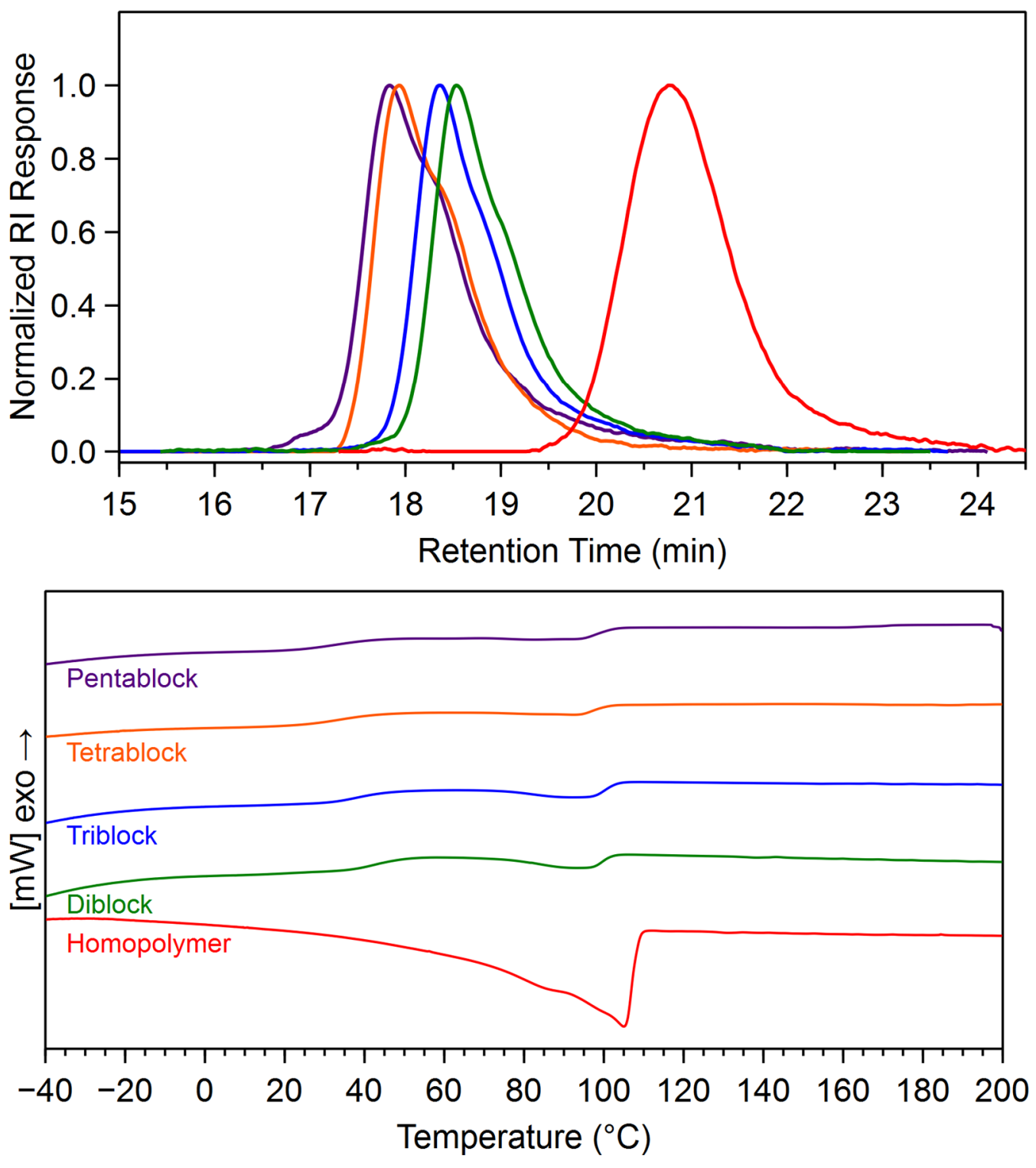

\begin{tabular}{ccccccccc}
\hline Sample & $\begin{array}{c}\text { Time } \\
(\mathbf{h})\end{array}$ & $\begin{array}{c}\text { Yield } \\
(\mathbf{g})\end{array}$ & $\begin{array}{c}\boldsymbol{M}_{\mathbf{n}} \\
(\mathbf{k D a})\end{array}$ & $\boldsymbol{D}$ & $\begin{array}{c}\boldsymbol{T}_{\mathbf{m}} \\
\left({ }^{\circ} \mathbf{C}\right)\end{array}$ & $\begin{array}{c}\Delta \mathbf{H} \\
(\mathbf{J} / \mathbf{g})\end{array}$ & $\begin{array}{c}\boldsymbol{X} \\
(\boldsymbol{\%})\end{array}$ & $\begin{array}{c}\text { Branches/ } \\
\mathbf{1 0 0 0 C}\end{array}$ \\
\hline Homopolymer & 2.0 & 0.022 & 9.1 & 1.34 & 105 & 113.2 & 38.6 & $\mathrm{n} / \mathrm{d}$ \\
Diblock & 0.7 & 0.070 & 56.9 & 1.36 & 37,96 & $3.0,7.5$ & 3.6 & $\mathrm{n} / \mathrm{d}$ \\
Triblock & 3.0 & 0.080 & 65.8 & 1.38 & 32,96 & $2.1,9.1$ & 3.8 & $\mathrm{n} / \mathrm{d}$ \\
Tetrablock & 0.7 & 0.106 & 99.0 & 1.34 & 28,93 & $1.1,5.3$ & 2.2 & $\mathrm{n} / \mathrm{d}$ \\
Pentablock & 5.0 & 2.97 & 106.5 & 1.39 & 25,94 & $1.8,6.4$ & 2.8 & 68 \\
\hline
\end{tabular}


Pentablock Copolymer GPC/DSC (Table 2, Entry 3)
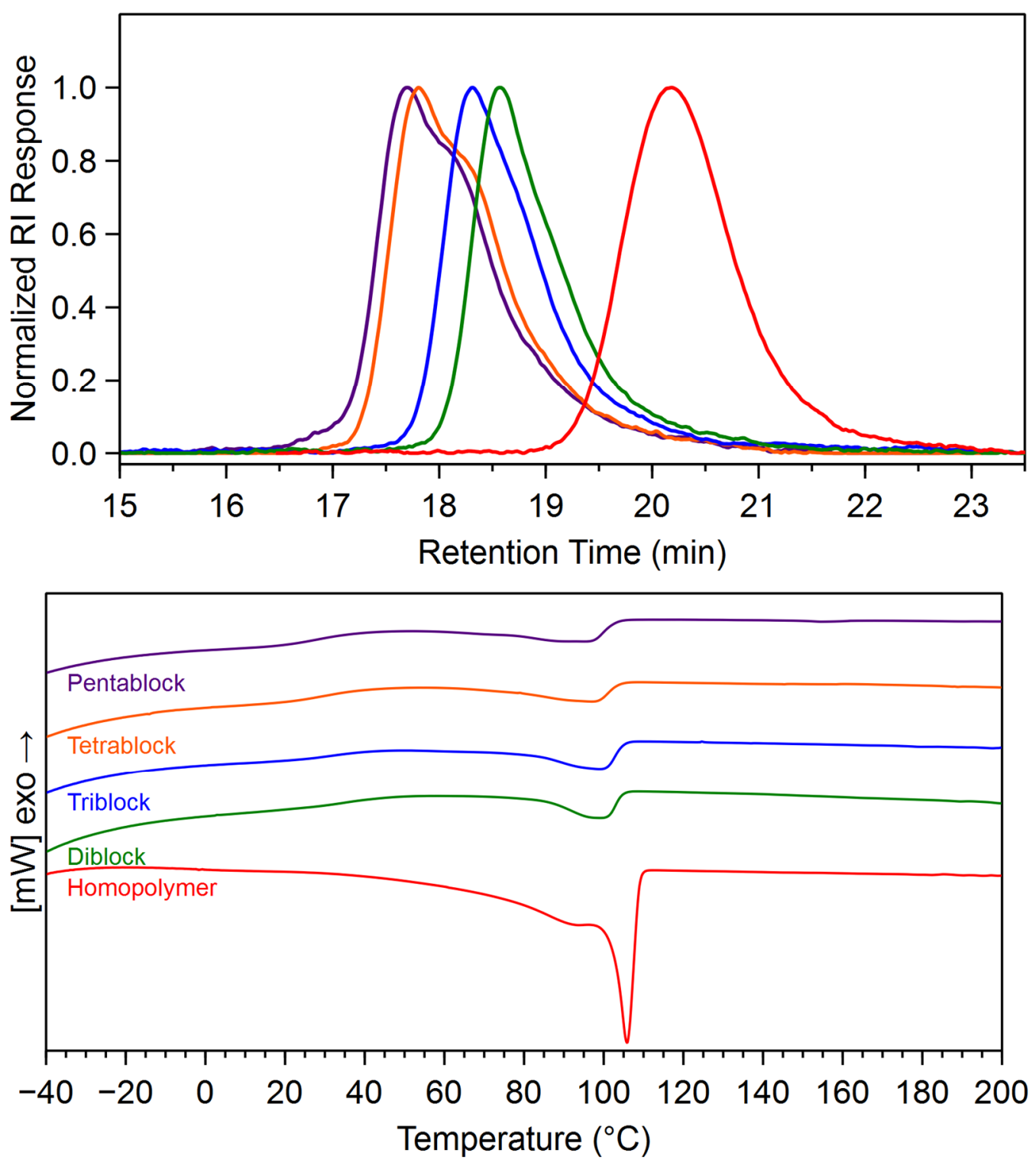

\begin{tabular}{ccccccccc}
\hline Sample & $\begin{array}{c}\text { Time } \\
(\mathbf{h})\end{array}$ & $\begin{array}{c}\text { Yield } \\
(\mathbf{g})\end{array}$ & $\begin{array}{c}\boldsymbol{M}_{\mathbf{n}} \\
(\mathbf{k D a})\end{array}$ & $\boldsymbol{D}$ & $\boldsymbol{T}_{\mathbf{m}}\left({ }^{\circ} \mathbf{C}\right)$ & $\begin{array}{c}\Delta \mathbf{H} \\
(\mathbf{J} / \mathbf{g})\end{array}$ & $\begin{array}{c}\boldsymbol{X} \\
(\boldsymbol{\%})\end{array}$ & $\begin{array}{c}\text { Branches/ } \\
\mathbf{1 0 0 0 C}\end{array}$ \\
\hline Homopolymer & 4.0 & 0.052 & 15.7 & 1.31 & 106 & 99.3 & 33.8 & 24 \\
Diblock & 0.7 & 0.069 & 58.6 & 1.31 & 30,100 & $0.8,14.4$ & 5.2 & $\mathrm{n} / \mathrm{d}$ \\
Triblock & 6.0 & 0.068 & 77.4 & 1.27 & 100 & 15.0 & 5.1 & $\mathrm{n} / \mathrm{d}$ \\
Tetrablock & 0.7 & 0.076 & 102.2 & 1.40 & 23,98 & $1.3,11.7$ & 4.4 & $\mathrm{n} / \mathrm{d}$ \\
Pentablock & 10.0 & 3.24 & 116.4 & 1.40 & 22,96 & $1.2,12.5$ & 4.7 & 64 \\
\hline
\end{tabular}


Heptablock Copolymer GPC/DSC (Table 2, Entry 4)
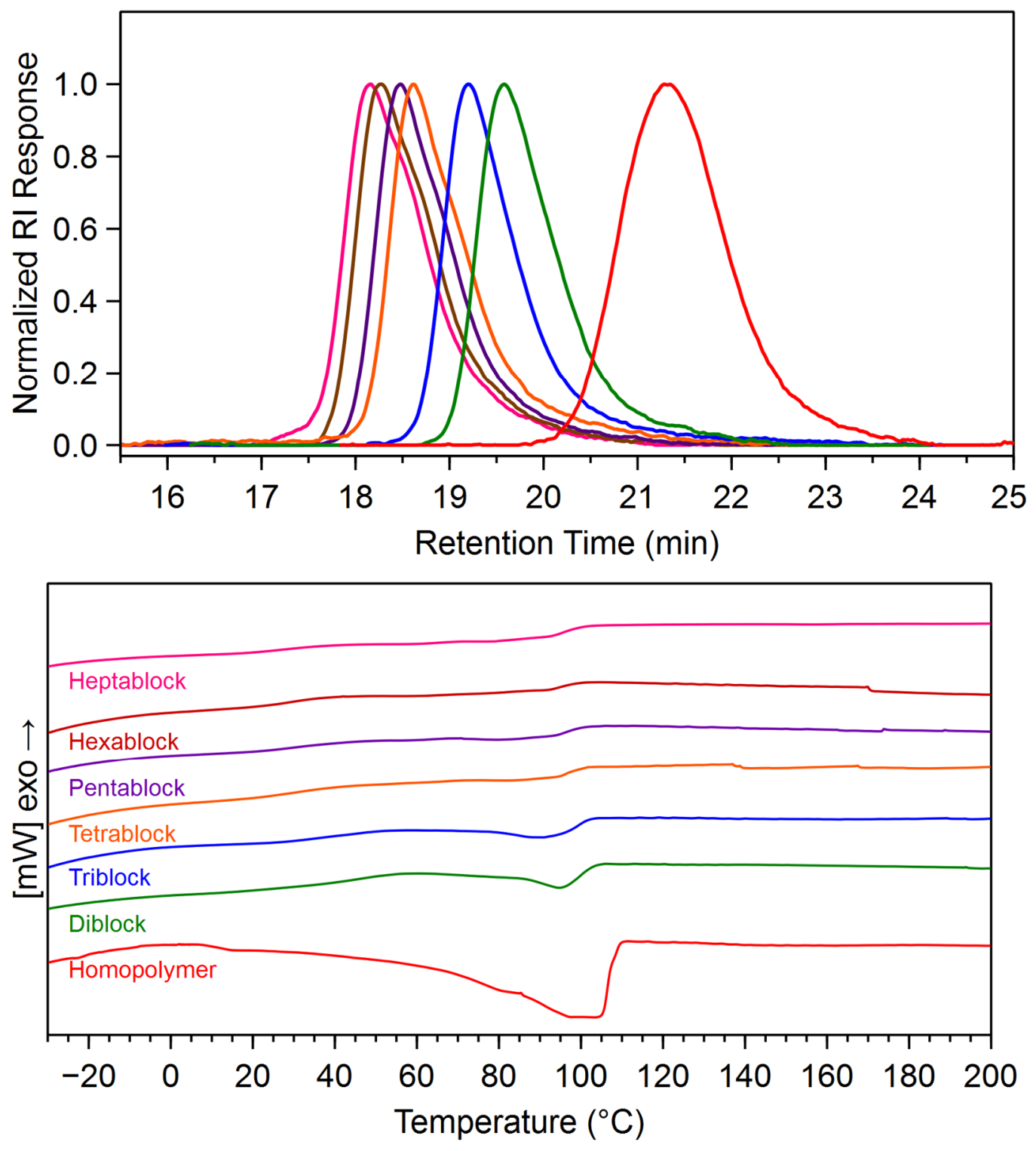

\begin{tabular}{ccccccccc}
\hline Sample & $\begin{array}{c}\text { Time } \\
(\mathbf{h})\end{array}$ & $\begin{array}{c}\text { Yield } \\
(\mathbf{g})\end{array}$ & $\begin{array}{c}\boldsymbol{M}_{\mathbf{n}} \\
(\mathbf{k D a})\end{array}$ & $\boldsymbol{D}$ & $\begin{array}{c}\boldsymbol{T}_{\mathbf{m}} \\
\left({ }^{\circ} \mathbf{C}\right)\end{array}$ & $\begin{array}{c}\Delta \mathbf{H} \\
(\mathbf{J} / \mathbf{g})\end{array}$ & $\begin{array}{c}\boldsymbol{X} \\
(\boldsymbol{\%})\end{array}$ & $\begin{array}{c}\text { Branches/ } \\
\mathbf{1 0 0 0 C}\end{array}$ \\
\hline Homopolymer & 1.5 & 0.011 & 5.6 & 1.33 & 103 & 109.0 & 37.1 & $\mathrm{n} / \mathrm{d}$ \\
Diblock & 0.3 & 0.029 & 23.9 & 1.30 & 41,95 & $1.4,12.5$ & 4.7 & $\mathrm{n} / \mathrm{d}$ \\
Triblock & 2.0 & 0.028 & 35.2 & 1.26 & 33,91 & $1.2,9.5$ & 3.6 & $\mathrm{n} / \mathrm{d}$ \\
Tetrablock & 0.3 & 0.044 & 55.1 & 1.32 & 93 & 6.2 & 2.1 & $\mathrm{n} / \mathrm{d}$ \\
Pentablock & 3.0 & 0.063 & 64.7 & 1.29 & 92 & 5.5 & 1.9 & $\mathrm{n} / \mathrm{d}$ \\
Hexablock & 0.4 & 0.064 & 77.0 & 1.29 & 92 & 3.6 & 1.2 & $\mathrm{n} / \mathrm{d}$ \\
Heptablock & 6.0 & 2.32 & 82.8 & 1.37 & 91 & 5.8 & 2.0 & 65 \\
\hline
\end{tabular}


Diblock Copolymer GPC/DSC (Table 3, Entry 1)
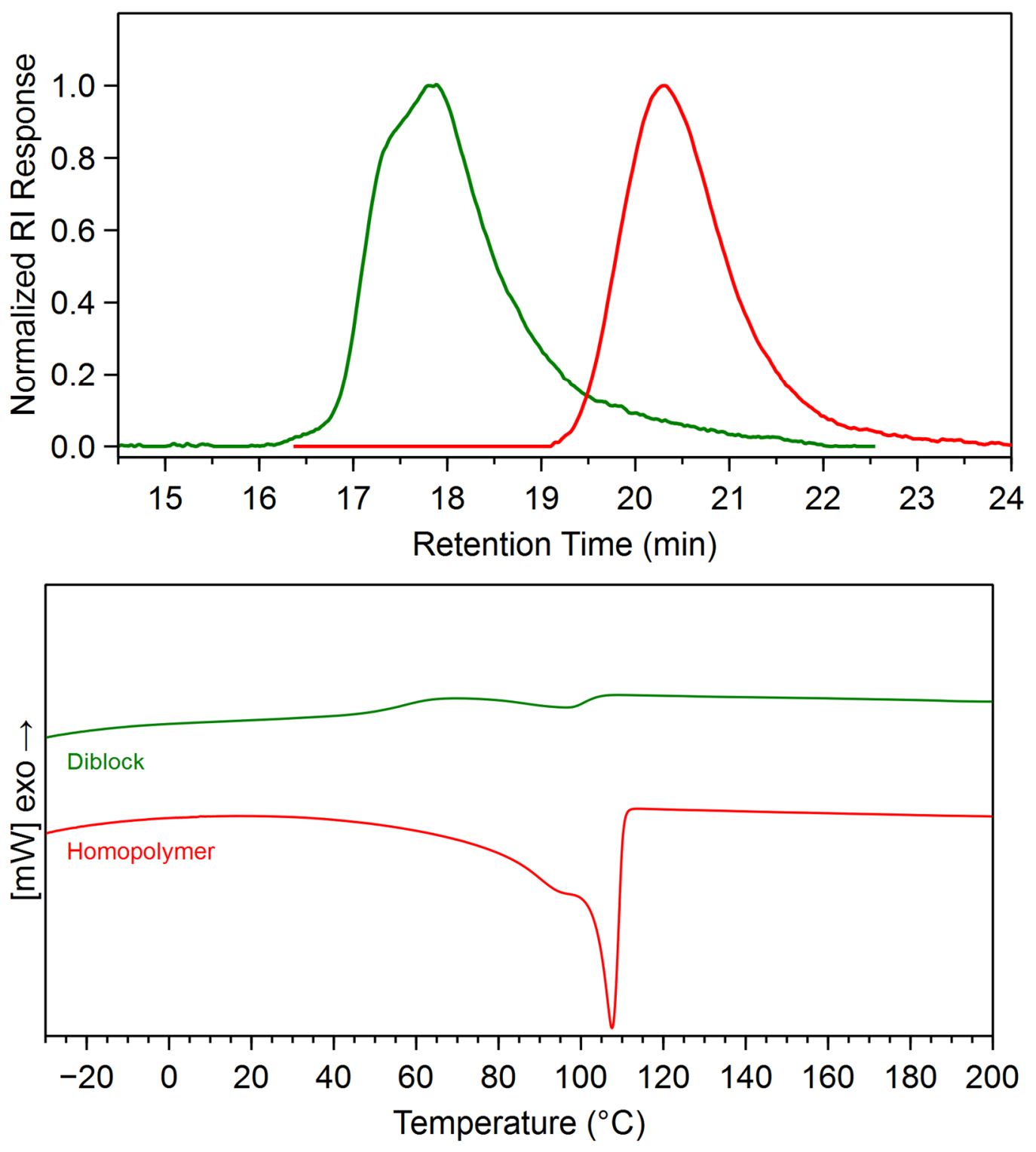

\begin{tabular}{ccccccccc}
\hline Sample & $\begin{array}{c}\text { Time } \\
(\mathbf{h})\end{array}$ & $\begin{array}{c}\text { Yield } \\
(\mathbf{g})\end{array}$ & $\begin{array}{c}\boldsymbol{M}_{\mathbf{n}} \\
(\mathbf{k D a})\end{array}$ & $\boldsymbol{D}$ & $\begin{array}{c}\boldsymbol{T}_{\mathbf{m}} \\
\left({ }^{\mathbf{C}} \mathbf{C}\right)\end{array}$ & $\begin{array}{c}\Delta \mathbf{H} \\
(\mathbf{J} / \mathbf{g})\end{array}$ & $\begin{array}{c}\boldsymbol{X} \\
(\boldsymbol{\%})\end{array}$ & $\begin{array}{c}\text { Branches/ } \\
\mathbf{1 0 0 0 C}\end{array}$ \\
\hline Homopolymer & 7.5 & 0.016 & 13.8 & 1.22 & 107 & 105.1 & 35.8 & $\mathrm{n} / \mathrm{d}$ \\
Diblock & 0.7 & 2.54 & 128.4 & 1.50 & 50,97 & $3.3,5.9$ & $1.1,2.0$ & 70 \\
\hline
\end{tabular}


Diblock Copolymer Data (Table 3, Entry 2)
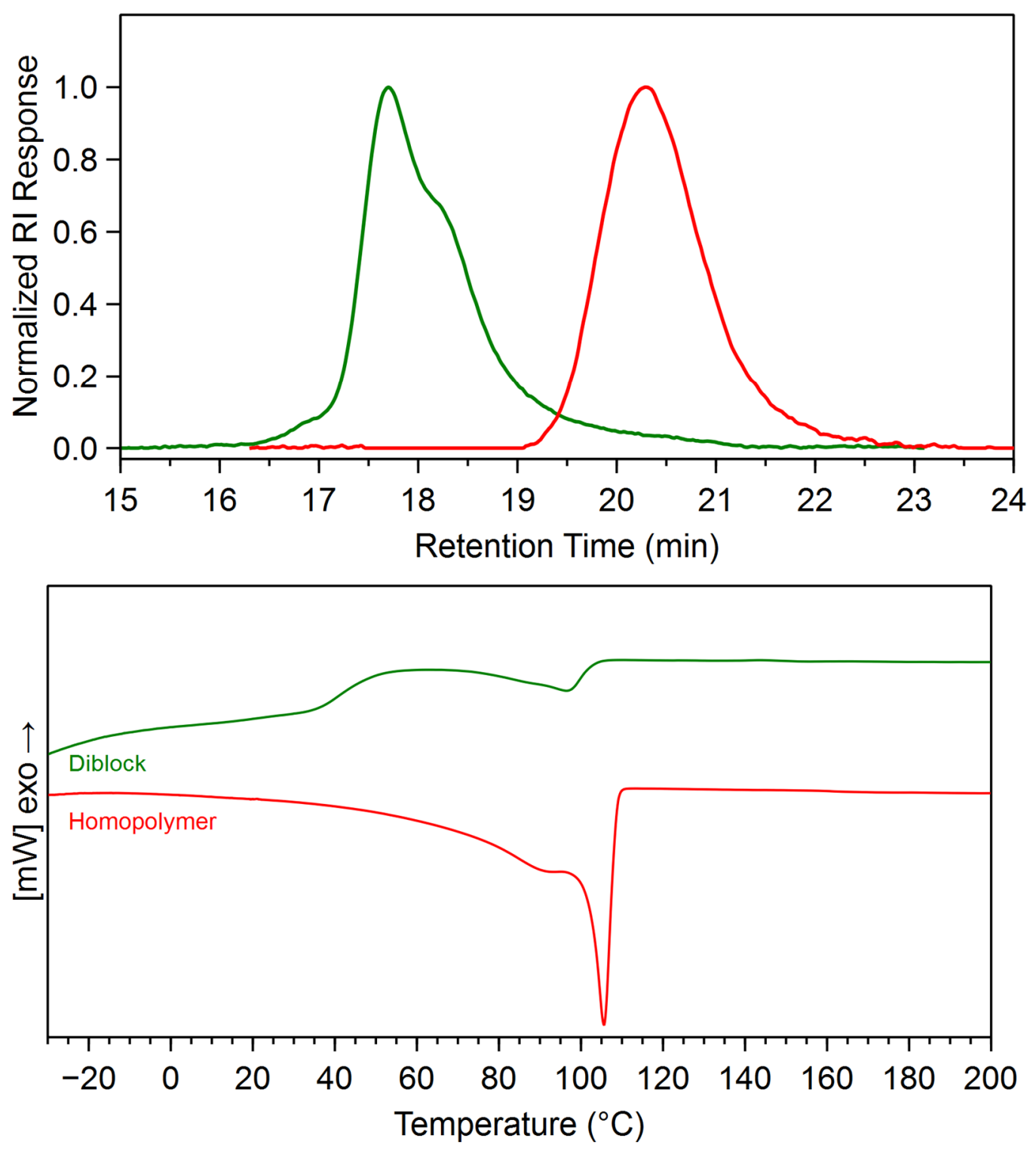

\begin{tabular}{ccccccccc}
\hline Sample & $\begin{array}{c}\text { Time } \\
(\mathbf{h})\end{array}$ & $\begin{array}{c}\text { Yield } \\
(\mathbf{g})\end{array}$ & $\begin{array}{c}\boldsymbol{M}_{\mathbf{n}} \\
(\mathbf{k D a})\end{array}$ & $\boldsymbol{D}$ & $\begin{array}{c}\boldsymbol{T}_{\mathbf{m}} \\
\left({ }^{\circ} \mathbf{C}\right)\end{array}$ & $\begin{array}{c}\Delta \mathbf{H} \\
(\mathbf{J} / \mathbf{g})\end{array}$ & $\begin{array}{c}\boldsymbol{X} \\
(\boldsymbol{\%})\end{array}$ & $\begin{array}{c}\text { Branches/ } \\
\mathbf{1 0 0 0 C}\end{array}$ \\
\hline Homopolymer & 3.5 & 0.020 & 15.2 & 1.27 & 106 & 118.5 & 40.4 & $\mathrm{n} / \mathrm{d}$ \\
Diblock & 1.5 & 2.43 & 121.9 & 1.38 & 36,97 & $4.0,6.5$ & $1.4,2.2$ & 72 \\
\hline
\end{tabular}


Diblock Copolymer Data (Table 3, Entry 3)
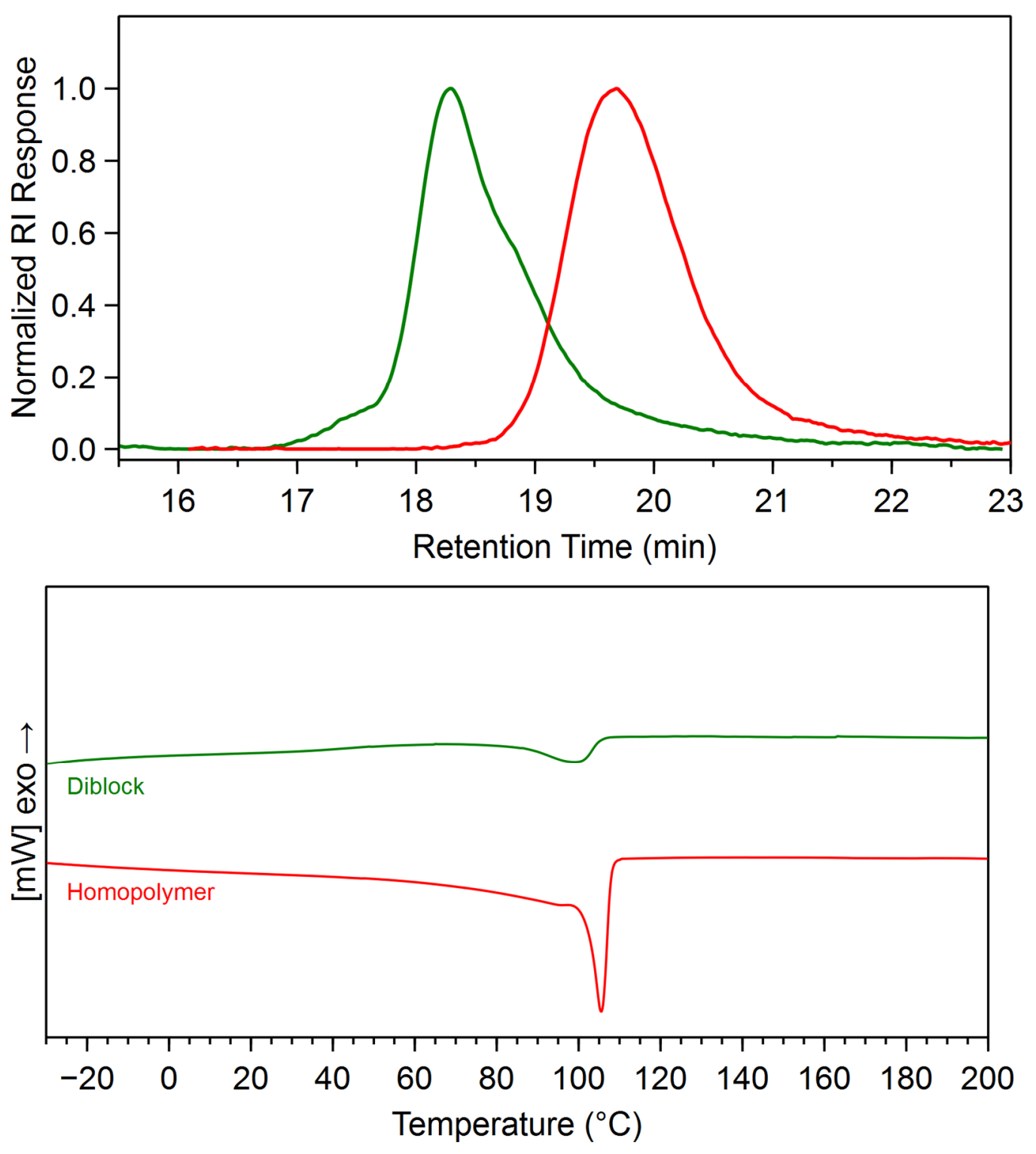

\begin{tabular}{ccccccccc}
\hline Sample & $\begin{array}{c}\text { Time } \\
(\mathbf{h})\end{array}$ & $\begin{array}{c}\text { Yield } \\
(\mathbf{g})\end{array}$ & $\begin{array}{c}\boldsymbol{M}_{\mathbf{n}} \\
(\mathbf{k D a})\end{array}$ & $\boldsymbol{D}$ & $\begin{array}{c}\boldsymbol{T}_{\mathbf{m}} \\
\left({ }^{\circ} \mathbf{C}\right)\end{array}$ & $\begin{array}{c}\Delta \mathbf{H} \\
(\mathbf{J} / \mathbf{g})\end{array}$ & $\begin{array}{c}\boldsymbol{X} \\
(\boldsymbol{\%})\end{array}$ & $\begin{array}{c}\text { Branches/ } \\
\mathbf{1 0 0 0 C}\end{array}$ \\
\hline Homopolymer & 8.0 & 0.032 & 26.6 & 1.22 & 106 & 106.0 & 36.1 & $\mathrm{n} / \mathrm{d}$ \\
Diblock & 0.3 & 1.93 & 85.2 & 1.27 & 100 & 16.0 & 5.4 & 62 \\
\hline
\end{tabular}


Statistical Copolymer Data (Table 3, Entry 4)
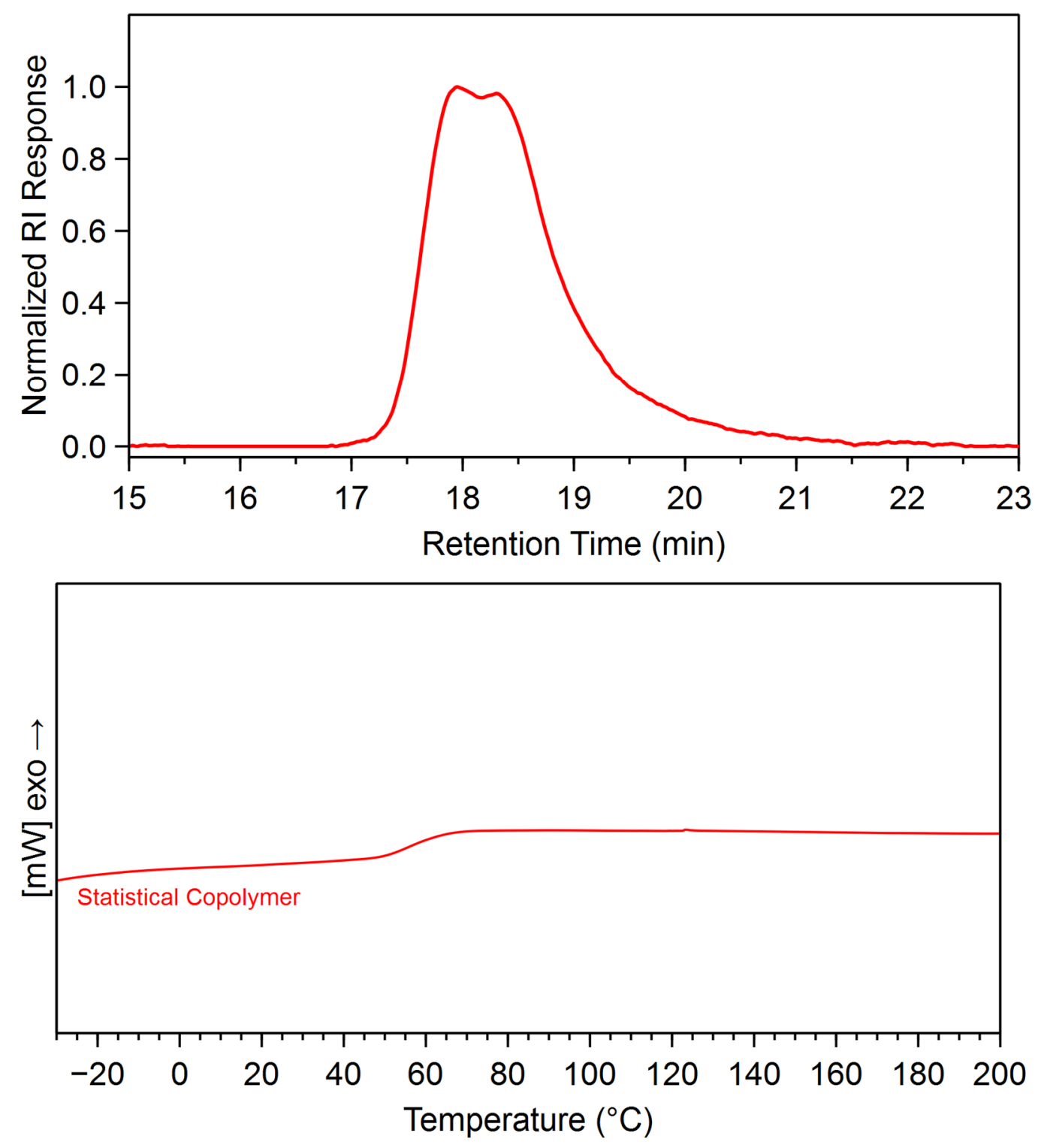

\begin{tabular}{ccccccccc}
\hline Sample & $\begin{array}{c}\text { Time } \\
(\mathbf{h})\end{array}$ & $\begin{array}{c}\text { Yield } \\
(\mathbf{g})\end{array}$ & $\begin{array}{c}\boldsymbol{M}_{\mathbf{n}} \\
(\mathbf{k D a})\end{array}$ & $\boldsymbol{D}$ & $\begin{array}{c}\boldsymbol{T}_{\mathbf{m}} \\
\left({ }^{\circ} \mathbf{C}\right)\end{array}$ & $\begin{array}{c}\Delta \mathbf{H} \\
(\mathbf{J} / \mathbf{g})\end{array}$ & $\begin{array}{c}\boldsymbol{X} \\
(\boldsymbol{\%})\end{array}$ & $\begin{array}{c}\text { Branches/ } \\
\mathbf{1 0 0 0 C}\end{array}$ \\
\hline Statistical & 1.0 & 2.33 & 102 & 1.3 & 49 & 6.4 & 2.2 & 72 \\
\hline
\end{tabular}


Statistical Copolymer Data (Table 3, Entry 5)
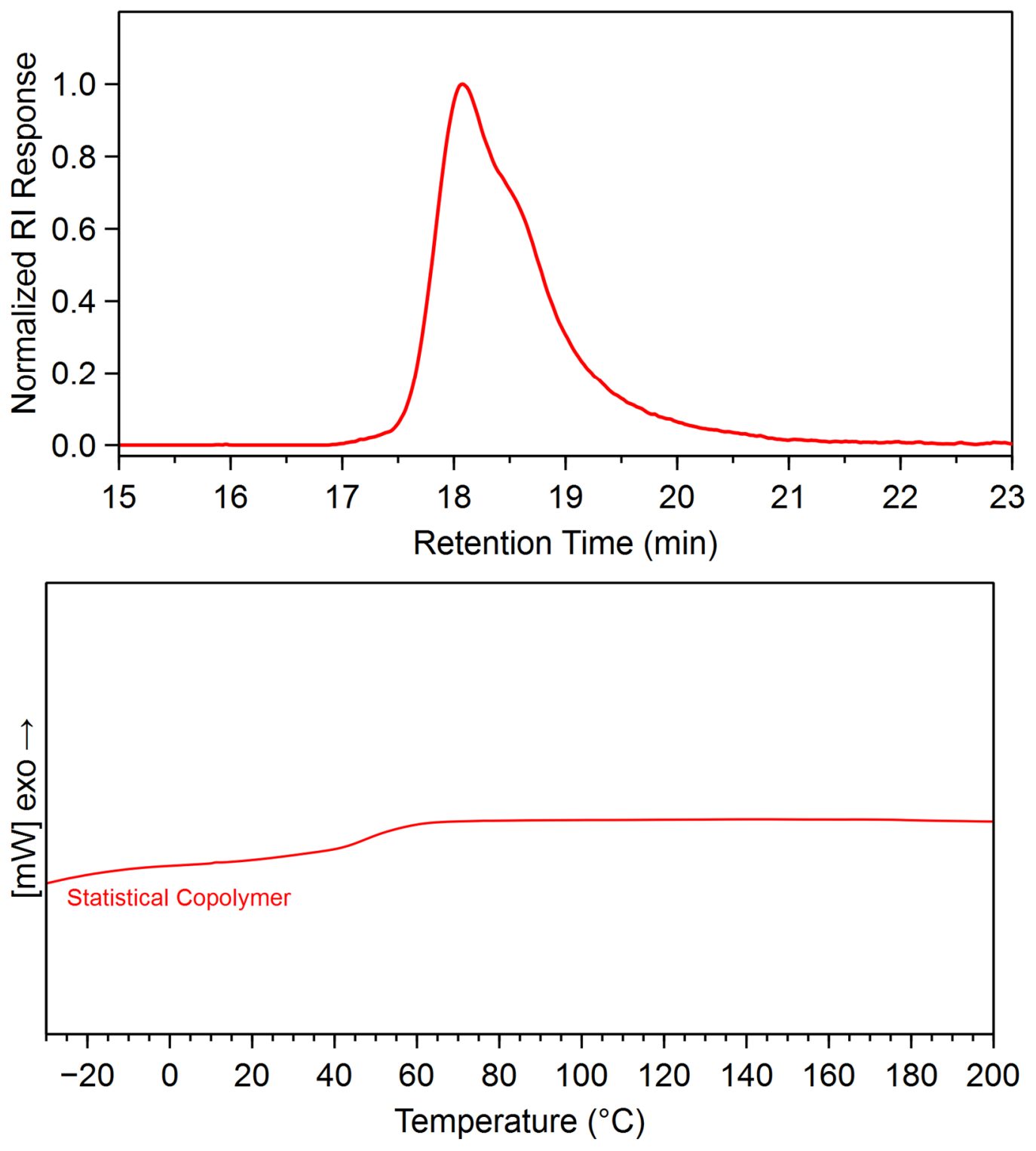

\begin{tabular}{ccccccccc}
\hline Sample & $\begin{array}{c}\text { Time } \\
(\mathbf{h})\end{array}$ & $\begin{array}{c}\text { Yield } \\
(\mathbf{g})\end{array}$ & $\begin{array}{c}\boldsymbol{M}_{\mathbf{n}} \\
(\mathbf{k D a})\end{array}$ & $\boldsymbol{D}$ & $\begin{array}{c}\boldsymbol{T}_{\mathbf{m}} \\
\left({ }^{\circ} \mathbf{C}\right)\end{array}$ & $\begin{array}{c}\Delta \mathbf{H} \\
(\mathbf{J} / \mathbf{g})\end{array}$ & $\begin{array}{c}\boldsymbol{X} \\
(\mathbf{\%})\end{array}$ & $\begin{array}{c}\text { Branches/ } \\
\mathbf{1 0 0 0 C}\end{array}$ \\
\hline Statistical & 2.0 & 3.44 & 102 & 1.2 & 41 & 4.0 & 1.4 & 83 \\
\hline
\end{tabular}




\section{Ethylene Homopolymerization}

A polymer sample was prepared using complex $\mathbf{2}$ in chlorobenzene with identical conditions to the statistical copolymer synthesis (page S5), except 1-decene was not included. The resulting polymer has similar properties compared to the statistical copolymer, suggesting negligible 1decene incorporation for the copolymerization of 1-decene and ethylene.
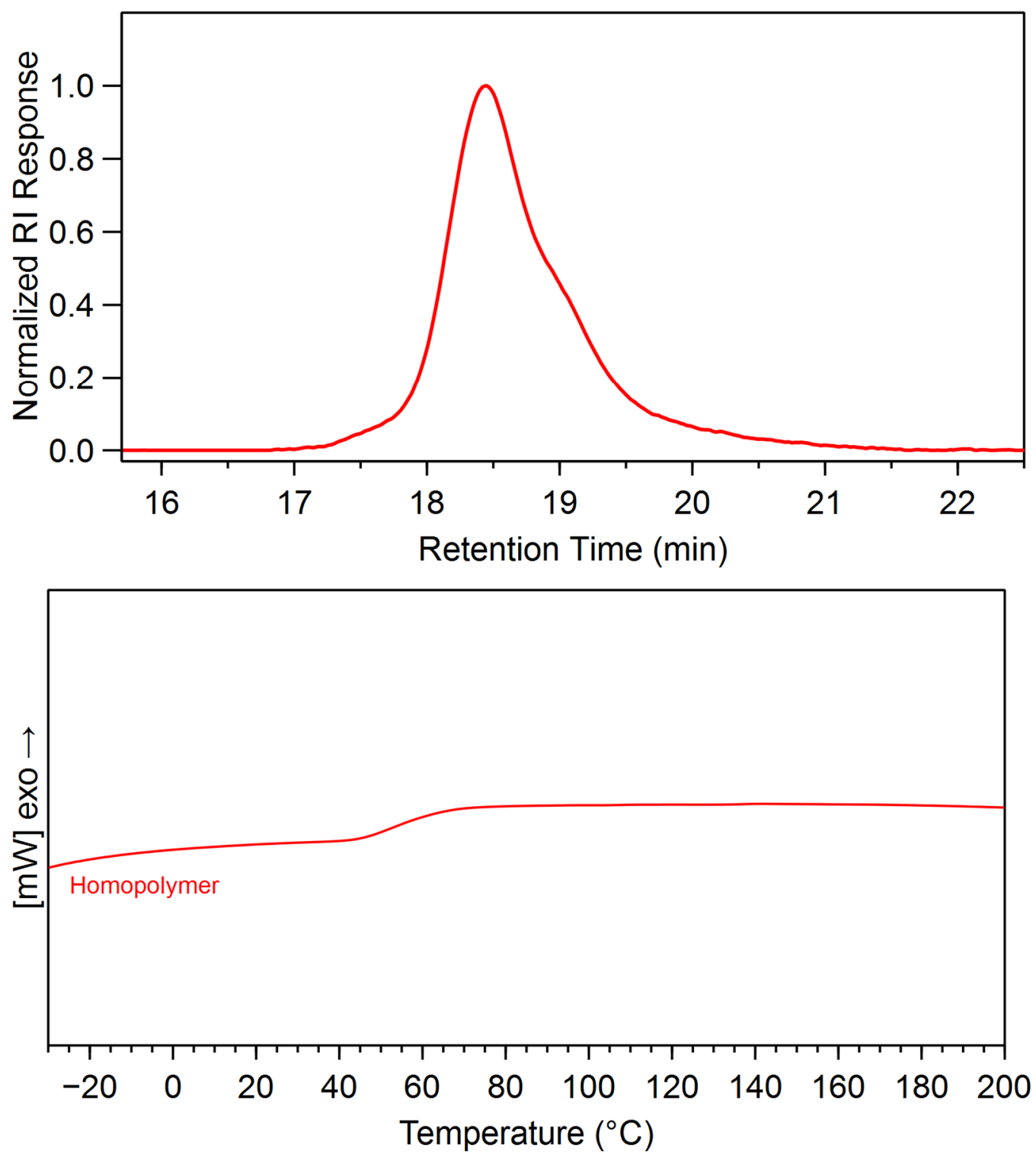

\begin{tabular}{ccccccccc}
\hline Sample & $\begin{array}{c}\text { Time } \\
(\mathbf{h})\end{array}$ & $\begin{array}{c}\text { Yield } \\
(\mathbf{g})\end{array}$ & $\begin{array}{c}\boldsymbol{M}_{\mathbf{n}} \\
(\mathbf{k D a})\end{array}$ & $\boldsymbol{D}$ & $\begin{array}{c}\boldsymbol{T}_{\mathbf{m}} \\
\left({ }^{\circ} \mathbf{C}\right)\end{array}$ & $\begin{array}{c}\Delta \mathbf{H} \\
(\mathbf{J} / \mathbf{g})\end{array}$ & $\begin{array}{c}\boldsymbol{X} \\
(\boldsymbol{\%})\end{array}$ & $\begin{array}{c}\text { Branches/ } \\
\mathbf{1 0 0 0 C}\end{array}$ \\
\hline Homopolymer & 1.5 & 2.11 & 125 & 1.28 & 46 & 4.9 & 1.7 & 77 \\
\hline
\end{tabular}




\section{Determination of Branching in Polymer Samples}

The number of branches per 1000 carbons (branches/1000C) for each final sample was determined using ${ }^{1} \mathrm{H}$ NMR as demonstrated by Rieger et. al. ${ }^{4}$ using the following equation:

$$
\text { total branching/1000C }=\frac{\frac{1}{3} I_{\mathrm{CH}_{3}}}{\frac{\left(I_{\mathrm{CH}_{2}}-\left(\frac{1}{3} I_{\mathrm{CH}_{3}}\right)\right)}{2}+\frac{1}{3} I_{\mathrm{CH}_{3}}} \times 1000
$$

Select samples were analyzed by quantitative ${ }^{13} \mathrm{C}$ NMR in order to determine the specific branching distribution. Signals were assigned based on previous literature reports. ${ }^{2,5}$ All signals were integrated, setting the total integral value to 1000 . Branching distributions were calculated based on the follow simplified equations below. Branching numbers determined between ${ }^{1} \mathrm{H}$ NMR and ${ }^{13} \mathrm{C}$ NMR are in agreement.

\begin{tabular}{|c|c|c|}
\hline $\begin{array}{c}\text { Signal } \\
\text { Number }\end{array}$ & $\begin{array}{c}\text { Chem. Shift } \\
(\mathrm{ppm})\end{array}$ & $\begin{array}{c}\text { Assignment } \\
(\text { see page } \mathrm{S} 31)\end{array}$ \\
\hline 1 & 13.33 & $1\left(1 \mathrm{~B}_{\mathrm{n}}\right)$ \\
\hline 2 & 19.27 & $1^{\prime}\left(1 \mathrm{~B}_{1}\right)$ \\
\hline 3 & 19.33 & $1^{\prime \prime}\left(1 \mathrm{~B}_{1}\right)$ \\
\hline 4 & 22.03 & $2\left(2 \mathrm{~B}_{\mathrm{n}}\right)$ \\
\hline 5 & 26.46 & $\beta\left(\beta \mathrm{B}_{\mathrm{n}}\right)$ \\
\hline 6 & 26.59 & $\beta^{\prime}\left(\beta \mathrm{B}_{1}\right)$ \\
\hline 7 & 26.99 & $\beta^{\prime \prime}\left(1,6-\beta^{\prime} \mathrm{B}_{\mathrm{n}}\right)$ \\
\hline 8 & 28.72 & $4\left(4 \mathrm{~B}_{\mathrm{n}}\right)$ \\
\hline 9 & 29.12 & $\delta, 5,6\left(\sigma \mathrm{B}_{1-\mathrm{n}}\right)$ \\
\hline 10 & 29.52 & $\gamma^{\prime}\left(\gamma \mathrm{B}_{1}\right)$ \\
\hline 11 & 29.62 & $\gamma\left(\gamma \mathrm{B}_{2-\mathrm{n}}\right)$ \\
\hline 12 & 31.36 & $3\left(3 \mathrm{~B}_{\mathrm{n}}\right)$ \\
\hline 13 & 32.44 & $\mathrm{M}^{\prime}\left(\mathrm{br}_{1} \mathrm{~B}_{1}\right)$ \\
\hline 14 & 32.76 & $\mathrm{M}^{\prime \prime}\left(1,4 / 6-\mathrm{brB}_{1}\right)$ \\
\hline 15 & 33.79 & $\alpha\left(\alpha \mathrm{B}_{\mathrm{n}}\right)$ \\
\hline 16 & 34.06 & $\alpha^{\prime \prime}\left(1,4 / 6-\alpha^{\prime} \mathrm{B}_{\mathrm{n}}\right)$ \\
\hline 17 & 36.73 & $\alpha^{\prime}\left(\alpha \mathrm{B}_{1}\right)$ \\
\hline 18 & 37.41 & $\mathrm{M}\left(\mathrm{br}_{3-\mathrm{n}}\right)$ \\
\hline
\end{tabular}

Methyl $=I_{1}+I_{1}{ }^{\prime}$

$$
\text { Hexyl }+=I_{2}+I_{3} / 2
$$

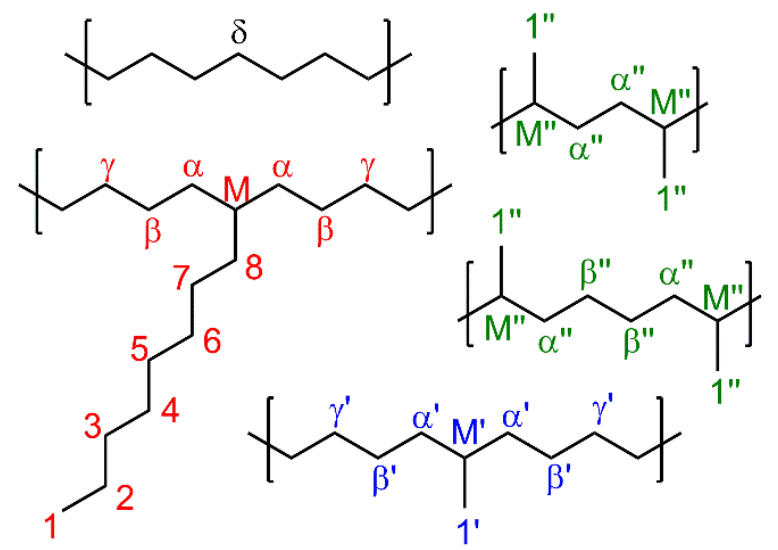




\section{Copies of ${ }^{1} \mathrm{H}$ NMR Spectra}

${ }^{1}$ H NMR of Representative Pentablock Copolymer (Table 2, Entry 1)

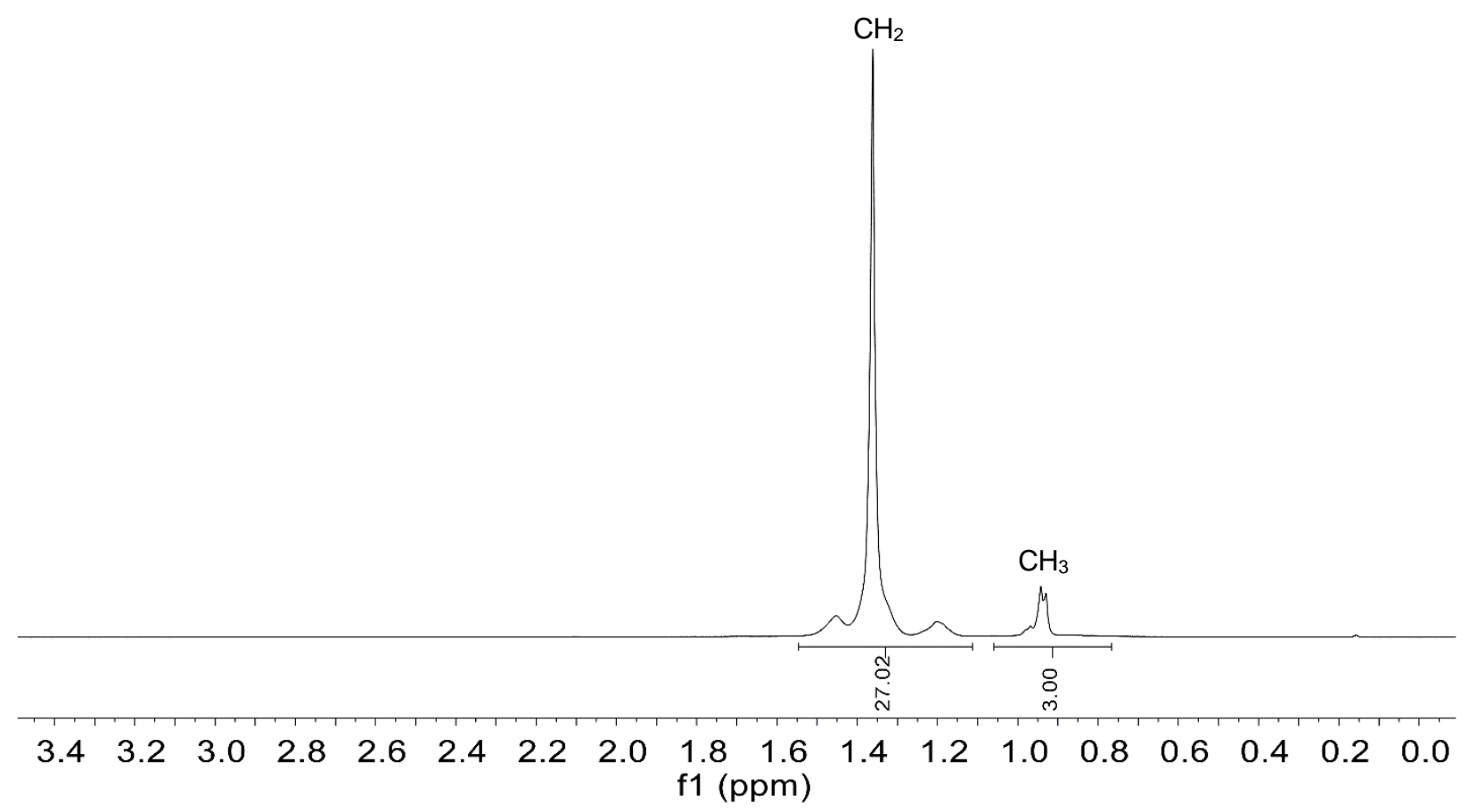

${ }^{1}$ H NMR of Representative Triblock Copolymer (Table 1, Entry 2)

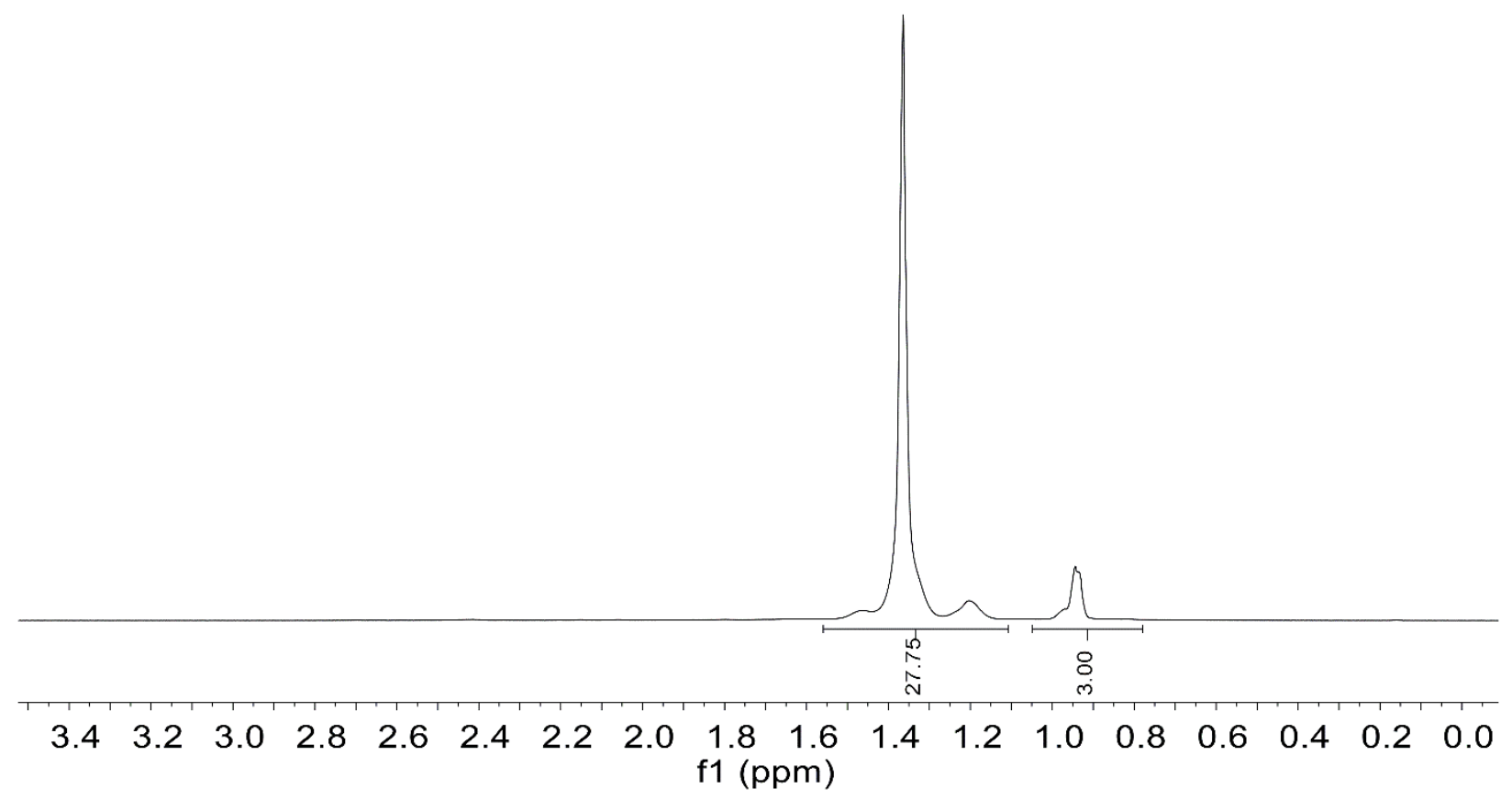


${ }^{1}$ H NMR of Representative Diblock Copolymer (Table 3, Entry 3)

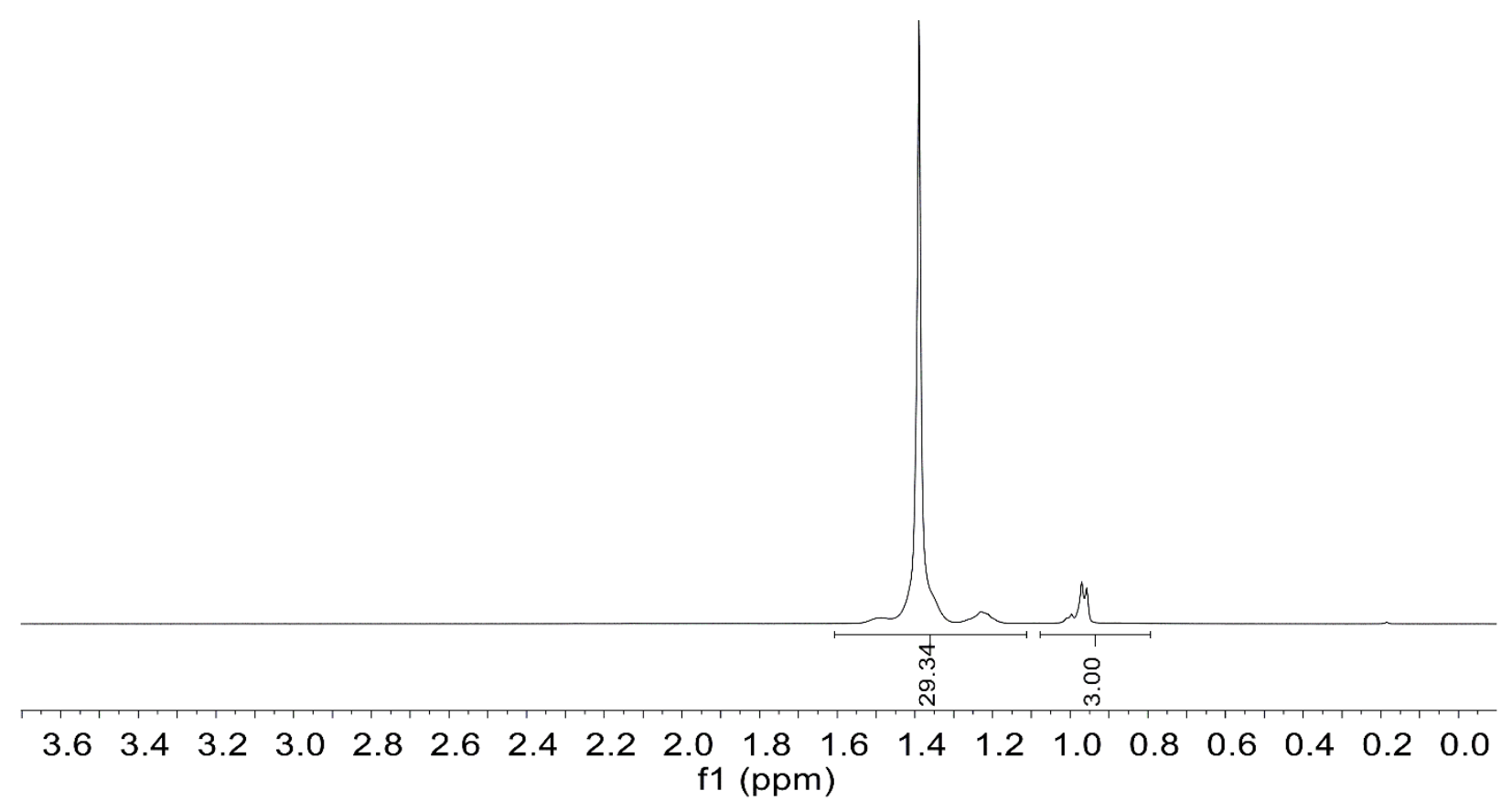

${ }^{1}$ H NMR of Representative Statistical Copolymer (Table 2, Entry 4)

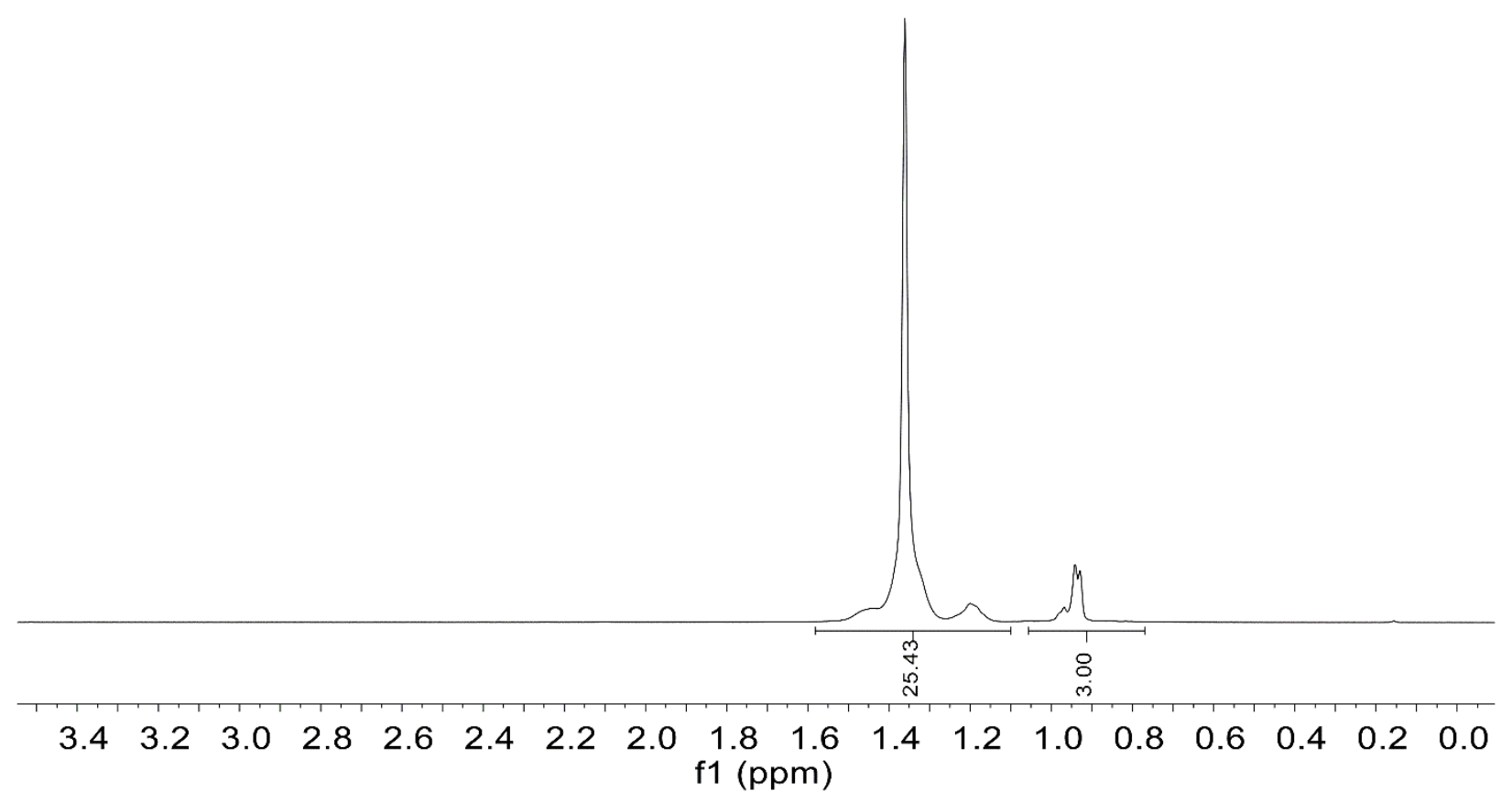




\section{2. ${ }^{13} \mathrm{C}$ NMR of Select Samples}

${ }^{13}$ C NMR Assignment of Triblock Copolymer (Table 1, Entry 6)

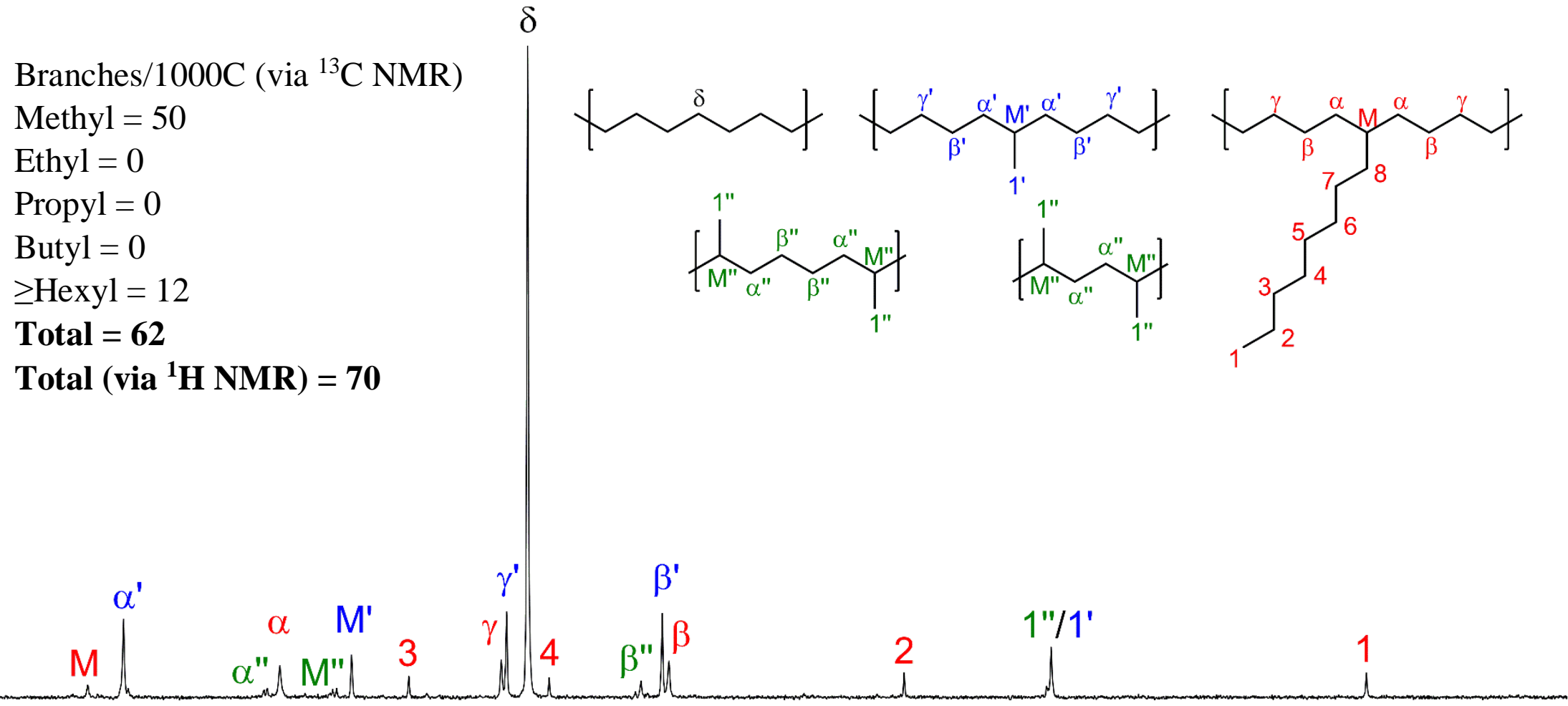

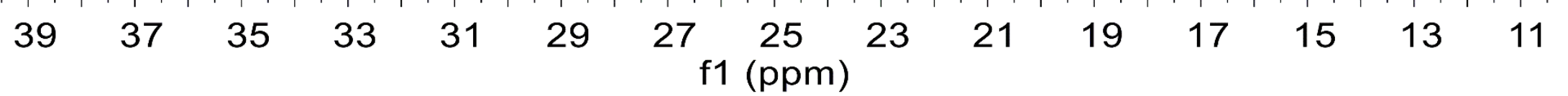


${ }^{13}$ C NMR of Representative Hard Block Homopolymer (Table 2, Entry 3, first block)

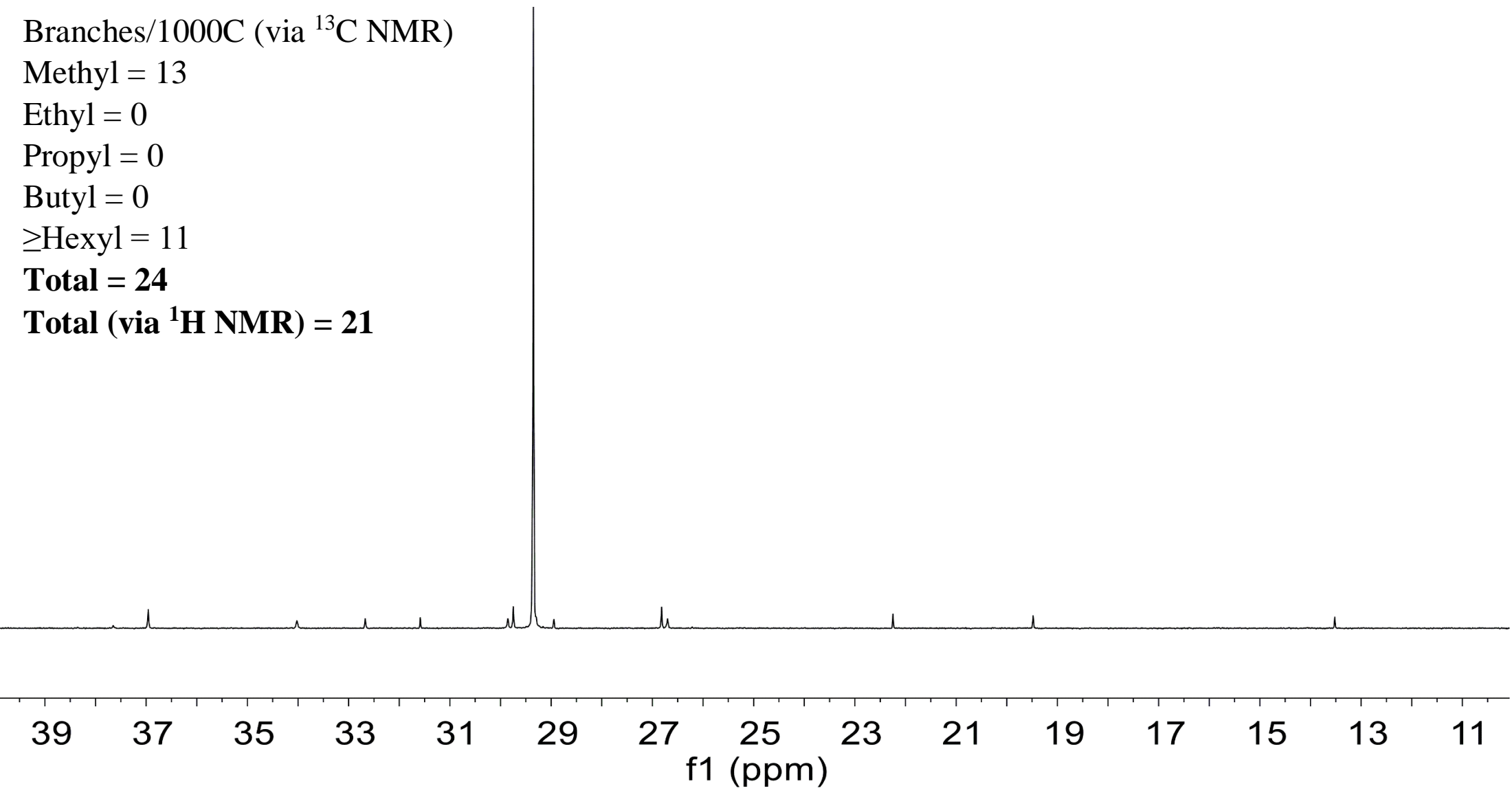


${ }^{13} \mathrm{C}$ NMR of Statistical Copolymer Grown in Chlorobenzene (soft block, $M_{\mathrm{n}}=91.8 \mathrm{kDa}, \oplus=1.3$ )

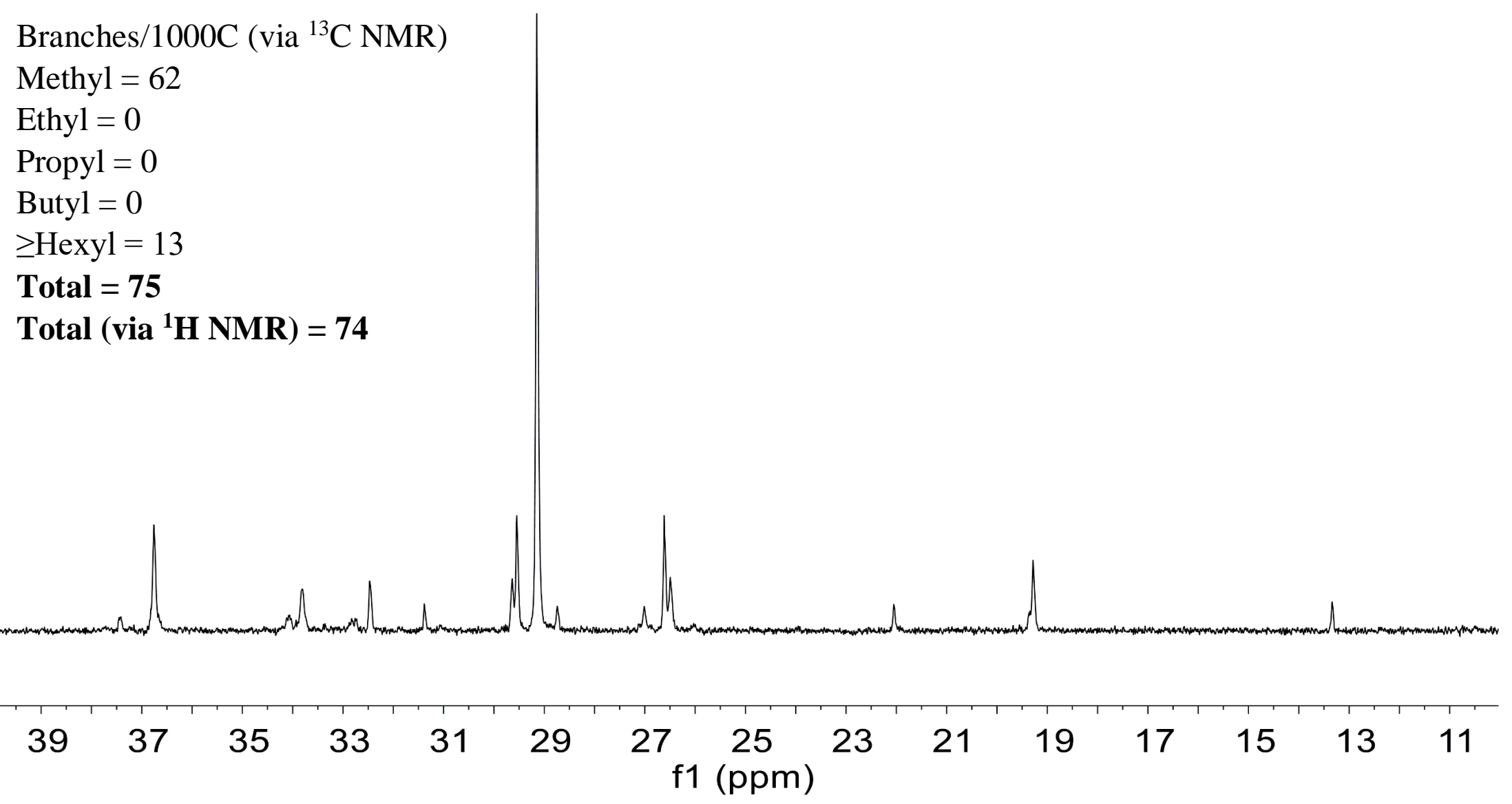




\section{Tensile Strength/Hysteresis Curves for Select Samples}

Pentablock Copolymer (Table 2, Entry 3)
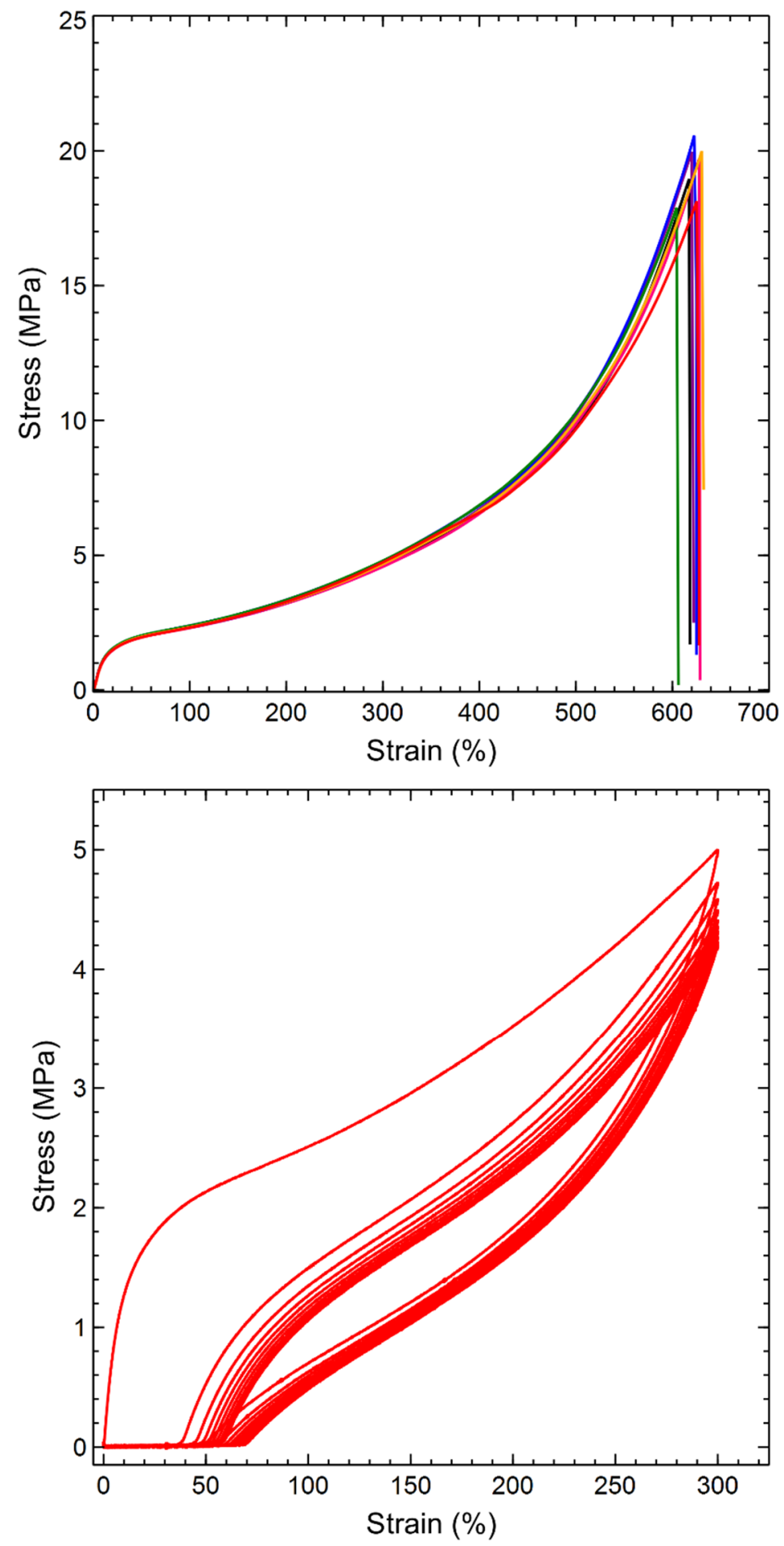
Triblock Copolymer (Table 1, Entry 6)
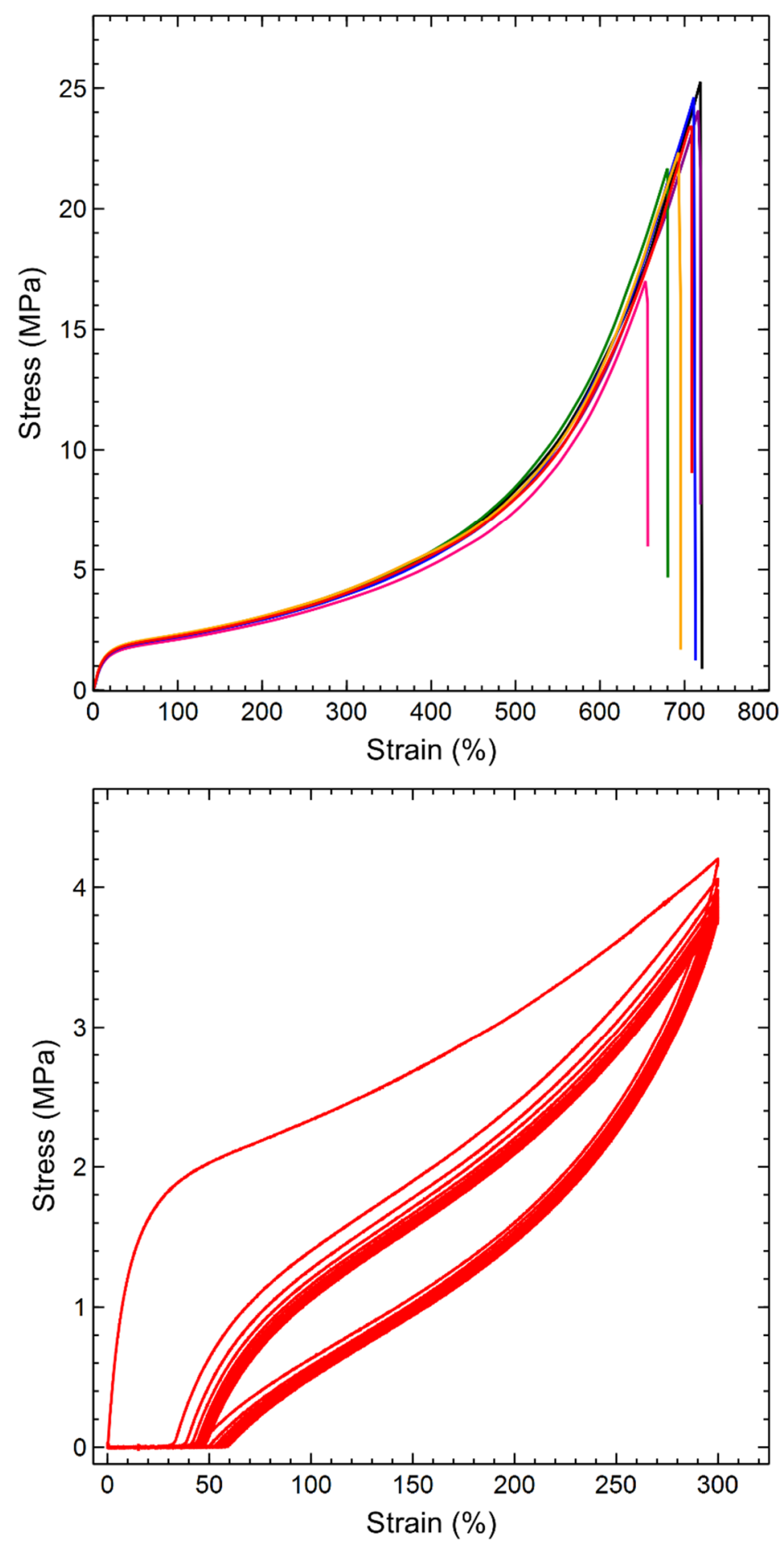
Diblock Copolymer (Table 3, Entry 3)
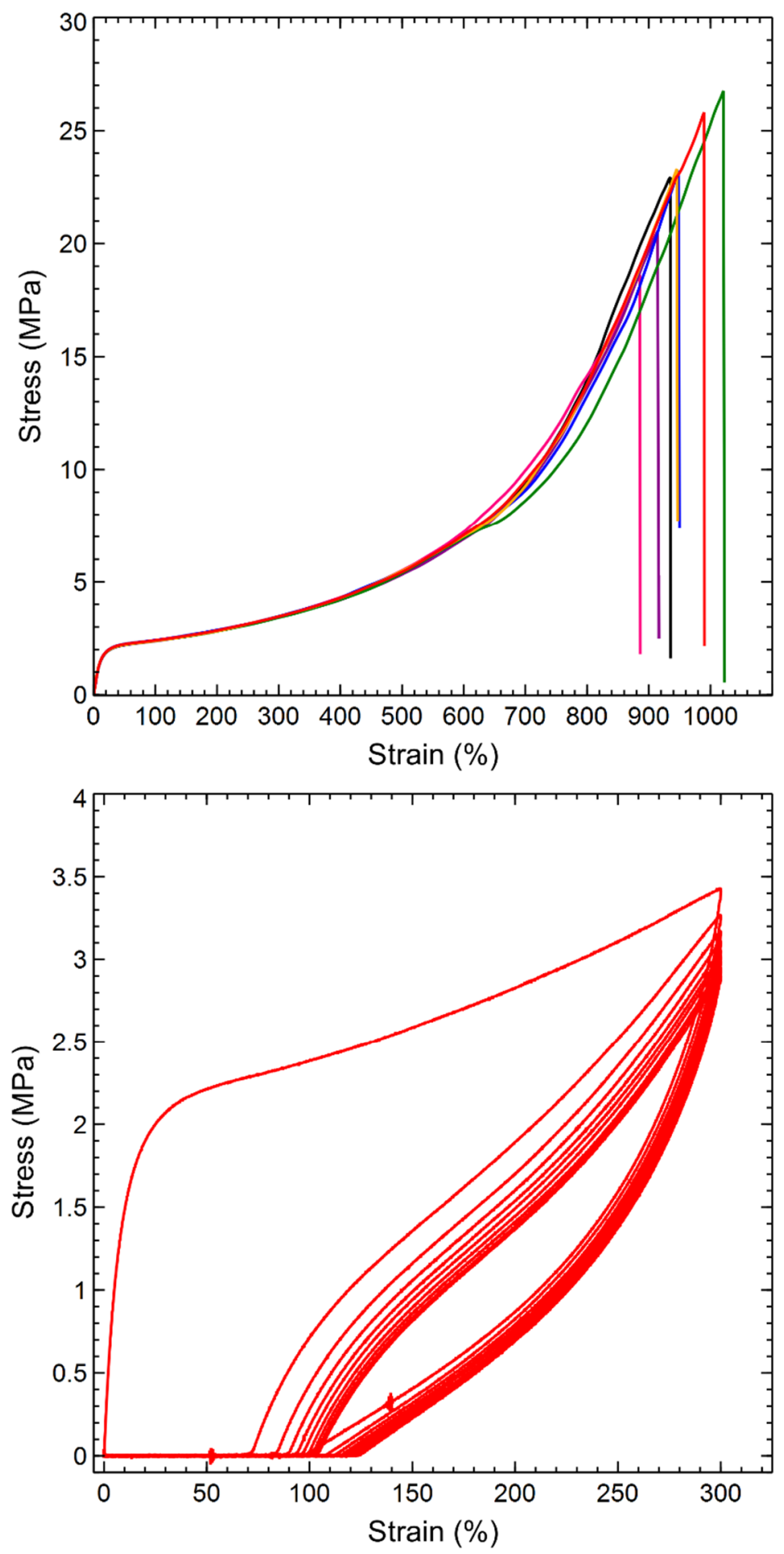
Statistical Copolymer Grown in Toluene (Table 3, Entry 4)
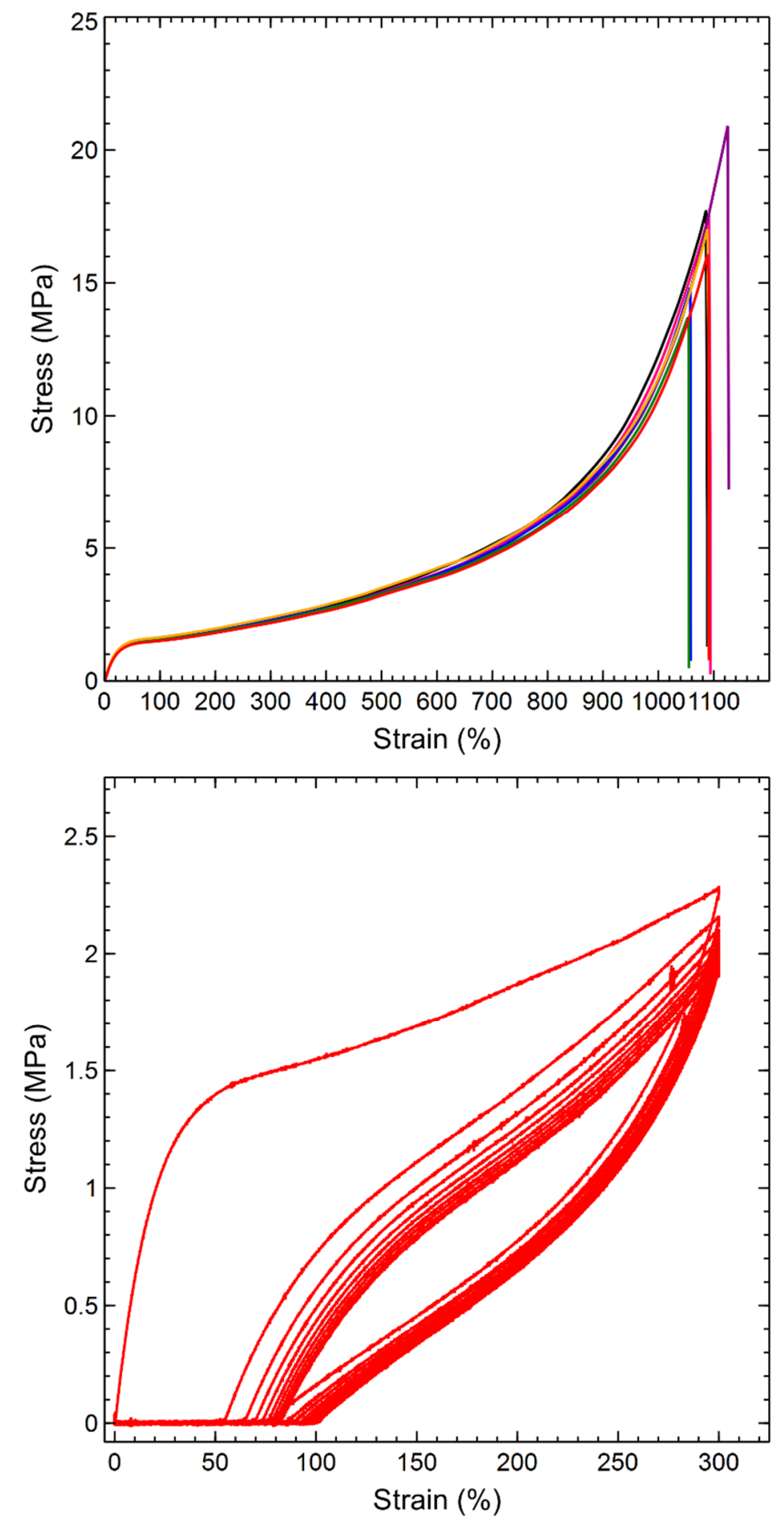
Statistical Copolymer Grown in Chlorobenzene (Table 3, Entry 5)
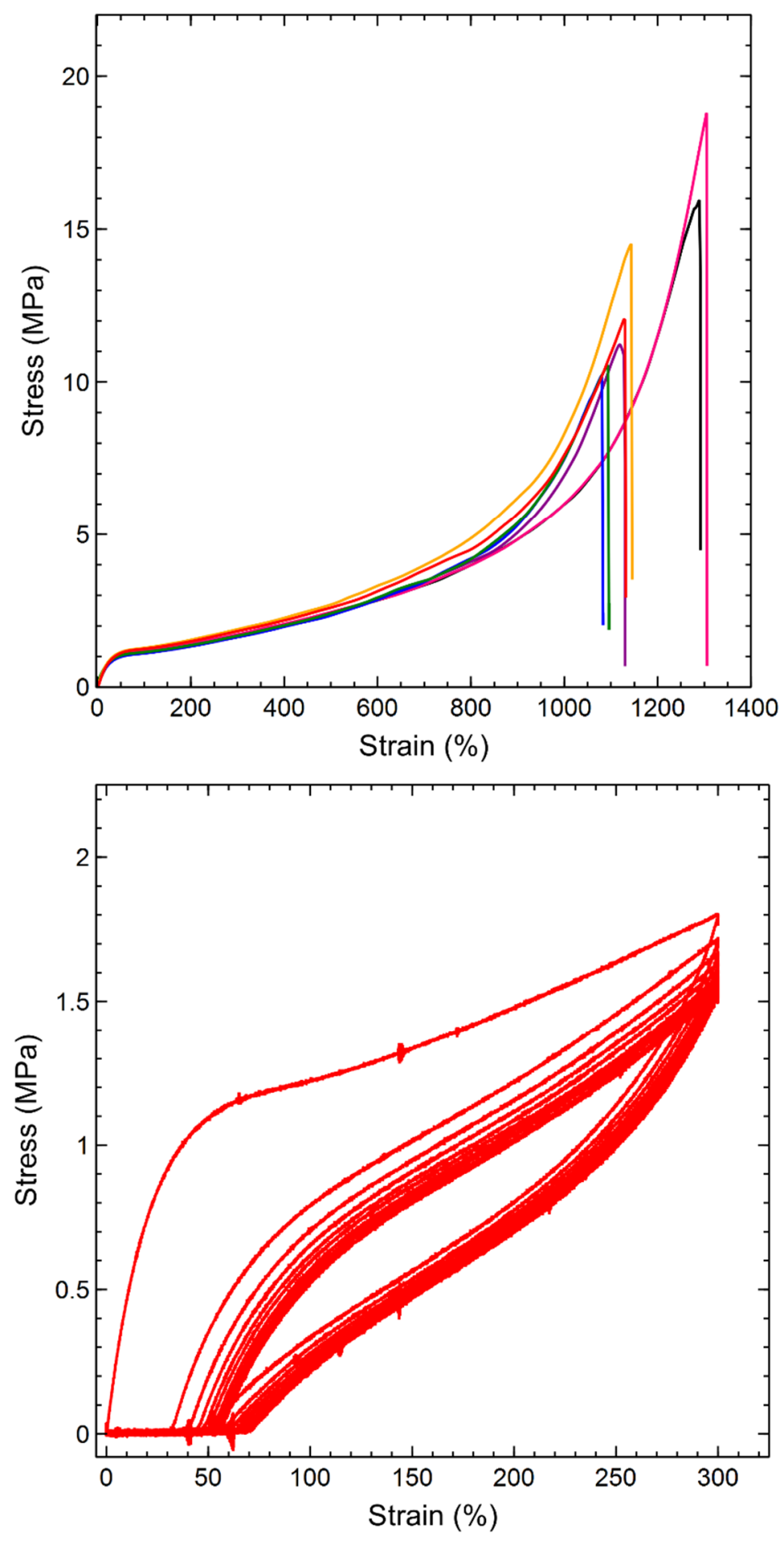


\section{Evidence of Strain-Induced Crystallization by DSC Analysis}

Two tensile bars cut from the same film (Table 3, entry 4) were analyzed and compared by DSC. One bar was left unstrained, while the other bar was rapidly stretched to $\sim 300 \%$ strain and relaxed approximately 15 times over the course of 30 seconds. A piece of the center gauge from each bar was cut using a razor blade and subjected to DSC measurements. The first heating traces were compared since we were specifically interested in sample history (strained vs unstrained). The unstrained sample shows a single major melting endotherm at $43{ }^{\circ} \mathrm{C}$, while the strained sample shows broadening, with two identifiable endotherms at $39^{\circ} \mathrm{C}$ and $46^{\circ} \mathrm{C}$. These results suggest that the crystallinity of the material changes after strain-induced deformation, potentially due to straininduced crystallization. Further studies need to be performed in order to better characterize this phenomenon.

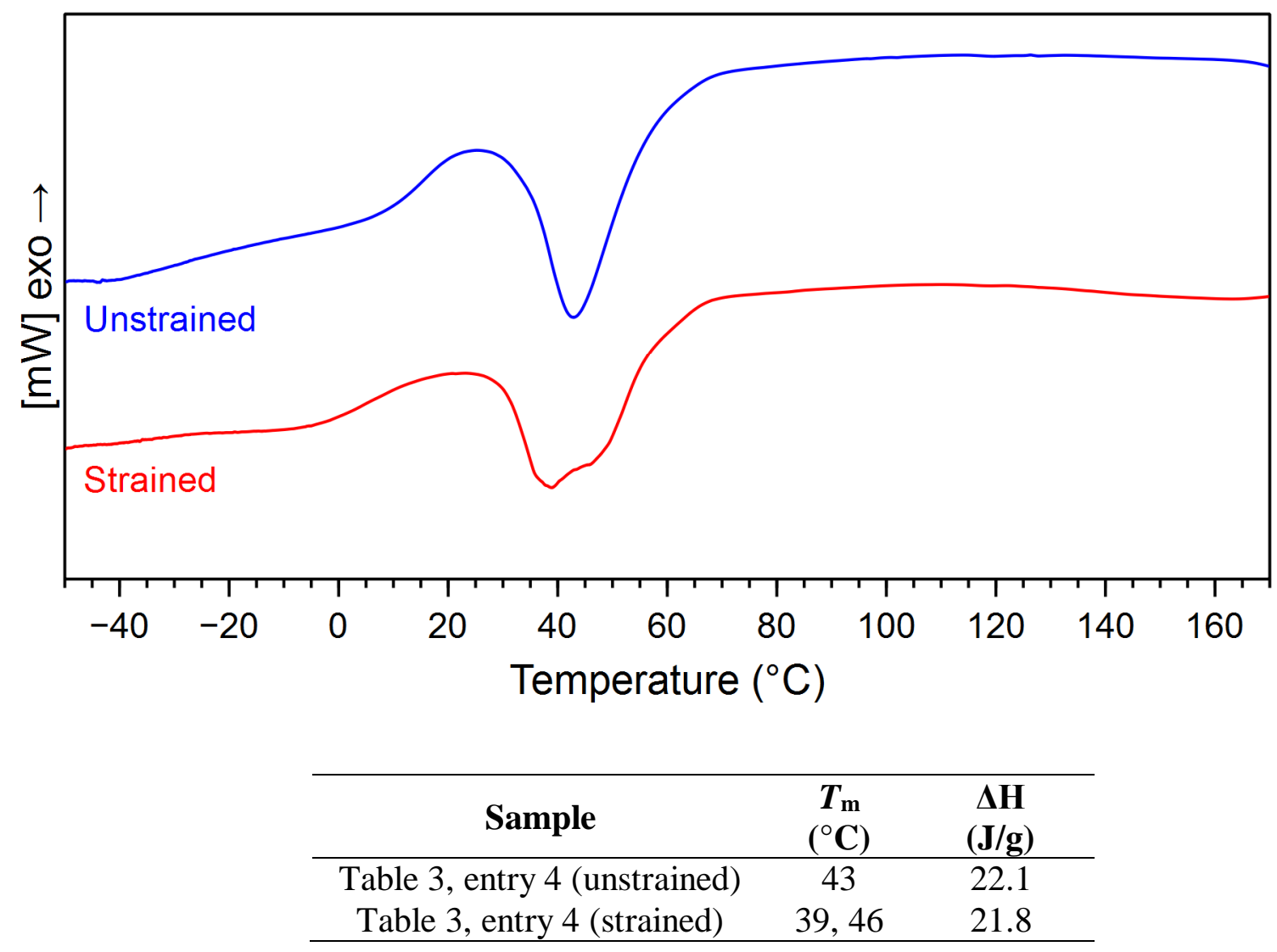




\section{References}

(1) Prasad, J. V.; Rao, P. V. C.; Garg, V. N. Quantification of Branching in Polyethylene by ${ }^{13}$ CNMR Using Paramagnetic Relaxation Agents. Eur. Polym. J. 1991, 27, 251-254.

(2) Vaidya, T.; Klimovica, K.; LaPointe, A. M.; Keresztes, I.; Lobkovsky, E. B.; Daugulis, O.; Coates, G. W. Secondary Alkene Insertion and Precision Chain-Walking: A New Route to Semicrystalline "Polyethylene" from $\alpha$-Olefins by Combining Two Rare Catalytic Events J. Am. Chem. Soc. 2014, 136, 7213-7216.

(3) Luo, X.; Xie, S.; Liu, J.; Hu, H.; Jiang, J.; Huang, W.; Gao, H.; Zhou, D.; Lü, Z.; Yan, D. The Relationship Between the Degree of Branching and Glass Transition Temperature of Branched Polyethylene: Experiment and Simulation. Polym. Chem. 2014, 5, 1305-1312.

(4) Meinhard D.; Wegner, M.; Kipiani, G.; Hearley, A.; Reuter, P.; Fischer, S.; Marti, O.; Rieger, B. New Nickel (II) Diimine Complexes and the Control of Polyethylene Microstructure by Catalyst Design. J. Am. Chem. Soc. 2007, 129, 9182-9191.

(5) Azoulay, J. D.; Bazan, G. C.; Galland, G. B. Microstructural Characterization of Poly(1-

hexene) Obtained Using a Nickel $\alpha$-Keto- $\beta$-diimine Initiator. Macromolecules 2010, 43, 2794-2800. 